\title{
INTEGRABILITY OF HAMILTONIAN SYSTEMS AND TRANSSERIES EXPANSIONS
}

\author{
WERNER BALSER AND MASAFUMI YOSHINO
}

\begin{abstract}
This paper studies analytic Liouville-non-integrable and $C^{\infty}$-Liouvilleintegrable Hamiltonian systems with two degrees of freedom. We will show that considerably general Hamiltonians than the one studied in [1] have the property. We also show that a certain monodromy property of an ordinary differential equation obtained as a subsystem of a given Hamiltonian and the transseries expansion of a first integral play an important role in the analysis. In the former half we will show that the analytic Liouville-non-integrability holds for a rather wider class of Hamiltonians than in [1] under a certain monodromy condition. For these analytic non integrable Hamiltonians we cannot construct nonanalytic first integrals concretely as in [1]. In the latter half, we show the nonanalytic integrability from the viewpoint of a transseries expansion of a first integral. We will construct a first integral in transseries formally under general situation. Then we discuss convergence or existence of the first integral which has a given formal transseries as an asymptotic expansion.
\end{abstract}

\section{INTRODUCTION}

A Hamiltonian system in $n$ degrees of freedom is said to be $C^{\infty}$-Liouville-integrable if there are $n$ smooth first integrals in involution which are functionally independent on an open dense set. If the first integrals are analytic, then we say that it is analyticLiouville-integrable. In the paper [1], Gorni and Zampieri showed the existence of a Hamiltonian system with two degrees of freedom which is not analytic-Liouvilleintegrable, while it is $C^{\infty}$-Liouville-integrable. The geometrical motivation to study such an example comes from the integrability of a geodesic flows and the Taímanov's problem. (cf. [1]). We note that the proof of analytic-nonintegrability relies on the power series expansion of a first integral, and the $C^{\infty}$ - integrability was proved by constructing concretely a smooth first integral. (cf. Remark after Corollary 2.2.) In this paper, we are interested in the analytical structures which yield nonintegrability in the framework of rather general Hamiltonians than those in previous works. In fact, we will show that the monodromy property of a certain subsystem of a given Hamiltonian plays an important role.

Date: July 26, 2009.

2000 Mathematics Subject Classification. Primary 35C10; Secondary 40G10, 35Q15 .

Key words and phrases. nonintegrability, Hamiltonian systems, transseries, summability.

Institut für Angewandte Analysis, Universität Ulm, D-89069 Ulm, Germany

Department of Mathematics, Graduate School of Science, Hiroshima University, 1-3-1 Kagamiyama, Higashi-Hiroshima 739-8526 Japan. 
For these analytic-nonintegrable Hamiltonians, we cannot construct a nonanalytic first integral concretely, and instead we make use of a transseries in order to construct such an integral. An integral in a transseries expansion is constructed via the Lagrange-Charpit system of a certain vector field obtained by restricting a given Hamiltonian vector field to an invariant manifold. The construction of a first integral as a formal transseries is elementary, while the convergence part is complicated due to the degeneracy of a given Hamiltonian. We will study the convergence from two different points of view, transformation of transseries and Borel summability argument for transseries.

This paper is organized as follows. In $\S 2$ we study the necessity for the analyticLiouville-nonintegrability under the monodromy condition. In $\S 3$ we give the proof of Theorem 2.1. In $\S 4$ we give the transseries expansion of the integrals in a formal sense. The convergence in a curved region and transformation of transseries is discussed in $\S 5$. In $\S 6$ we study the asymptotic property of transseries in terms of Borel summability method.

\section{Analytic nOnintegrability}

Let $\sigma \geq 1$ be an integer and let $r\left(q_{1}, q_{2}, p_{1}, p_{2}\right)$ be an analytic function of $\left(q_{1}, q_{2}, p_{1}, p_{2}\right) \in$ $\mathbb{R}^{4}$ in some neighborhood of the origin $0 \in \mathbb{R}^{4}$ such that

$$
r \equiv r\left(q_{1}, q_{2}, p_{1}, p_{2}\right)=q_{1}^{2 \sigma}+a\left(q_{1}^{2 \sigma}\right) q_{2}^{2}+\tilde{r}\left(q_{1}, q_{2}, p_{1}, p_{2}\right) q_{2}^{3},
$$

where $\tilde{r}\left(q_{1}, q_{2}, p_{1}, p_{2}\right)$ is analytic at the origin and $a(t)\left(t=q_{1}^{2 \sigma}\right)$ is a polynomial of $t$ such that $a(0)>0$. We are interested in the following analytic Hamiltonian in $\mathbb{R}^{4}$ with two degrees of freedom

$$
\mathcal{H}=-q_{2} p_{2} \partial_{q_{1}} r+\left(r^{2}+q_{2} \partial_{q_{2}} r\right) p_{1},
$$

where $\partial_{q_{1}}=\frac{\partial}{\partial q_{1}}$ and $\partial_{q_{2}}=\frac{\partial}{\partial q_{2}}$. The associated Hamiltonian system is given by

$$
\left\{\begin{array}{l}
\dot{q}_{1}=\partial \mathcal{H} /\left(\partial p_{1}\right)=r^{2}+q_{2} \partial_{q_{2}} r+\left(2 r \partial_{p_{1}} r+q_{2} \partial_{q_{2}} \partial_{p_{1}} r\right) p_{1}-q_{2} p_{2} \partial_{p_{1}} \partial_{q_{1}} r \\
\dot{q}_{2}=\partial \mathcal{H} /\left(\partial p_{2}\right)=-q_{2} \partial_{q_{1}} r-q_{2} p_{2} \partial_{q_{1}} \partial_{p_{2}} r+p_{1}\left(2 r \partial_{p_{2}} r+q_{2} \partial_{q_{2}} \partial_{p_{2}} r\right) \\
\dot{p}_{1}=-\partial \mathcal{H} /\left(\partial q_{1}\right)=q_{2} p_{2} \partial_{q_{1}}^{2} r-\left(2 r \partial_{q_{1}} r+q_{2} \partial_{q_{1}} \partial_{q_{2}} r\right) p_{1} \\
\dot{p}_{2}=-\partial \mathcal{H} /\left(\partial q_{2}\right)=p_{2} \partial_{q_{1}} r+q_{2} p_{2} \partial_{q_{1}} \partial_{q_{2}} r-\left(2 r \partial_{q_{2}} r+\partial_{q_{2}} r+q_{2} \partial_{q_{2}}^{2} r\right) p_{1}
\end{array}\right.
$$

We need a definition in order to state our theorem.

DEFINITION 2.1. We say that a polynomial a(t) satisfies the monodromy condition $(M)$ if the following equation has no polynomial solution $U(t)$

$$
2 \sigma t^{2} U^{\prime}-4 \sigma U+(1-6 \sigma) t U=(t+1) a(t) .
$$

Then we have

THEOREM 2.1. Assume that a(t) satisfy (M). Then the Hamiltonian system (2.3) is not analytic-Liouville-integrable in any neighborhood of the origin. More precisely, for any analytic first integral $u=u\left(q_{1}, q_{2}, p_{1}, p_{2}\right)$ of (2.3) in $\mathbb{R}^{4}$, there exists a function $\phi$ of one-variable, being analytic at $0 \in \mathbb{R}$ such that $u=\phi \circ \mathcal{H}$. 
If $a(t) \equiv 1$, then we can easily see, from the direct computations or by Lemma 2 of [4] that (M) is satisfied. Hence we have

Corollary 2.2. Suppose that $\sigma=1, a(t) \equiv 1$ and $\tilde{r} \equiv 0$ in (2.1). Then the Hamiltonian system (2.3) is not analytic-Liouville-integrable in any neighborhood of the origin.

Remark. (a) As to the fundamental properties of (M) we refer [4]. In this paper, we change the terminology for the sake of simplicity. We remark that $(\mathrm{M})$ is a generic condition.

(b) Theorem 2.1 is a generalization of [4, Theorem 1], where the function $r$ in (2.1) was supposed to be independent of $p_{1}$ and $p_{2}$. Corollary 2.2 was proved in [1]. In this case, it is not difficult to see that (2.3) in Corollary 2.2 is $C^{\infty}$-Liouville-integrable, because it has a smooth first integral

$$
u= \begin{cases}q_{2} \exp \left(-\frac{1}{r}\right) & \text { if }\left(q_{1}, q_{2}\right) \neq(0,0), \\ 0 & \text { if }\left(q_{1}, q_{2}\right)=(0,0) .\end{cases}
$$

On the other hand, it is not known whether (2.3) in a general case has a nonanalytic first integral because one cannot construct the first integral of (2.3) concretely since $r$ also depends on $p_{1}$ and $p_{2}$. In $\S 4$ we will study the integrability from the viewpoint of transseries.

\section{Proof of THEOREM}

The proof of Theorem 2.1 is done by the argument in [4]. For the sake of completeness we give the proof.

Proof of Theorem 2.1. By the suitable change of the variable $q_{2}$ one may assume that $a(0)=1$. Let $u=u\left(q_{1}, q_{2}, p_{1}, p_{2}\right)$ be any analytic first integral of (2.3). We note that $u$ is the first integral of the Hamiltonian system (2.3) if and only if $u$ is a solution of the following first order equation

$$
\begin{gathered}
\{\mathcal{H}, u\} \equiv\left(q_{2} p_{2} \partial_{q_{1}}^{2} r-\left(2 r \partial_{q_{1}} r+q_{2} \partial_{q_{1}} \partial_{q_{2}} r\right) p_{1}\right) \frac{\partial u}{\partial p_{1}} \\
+\quad\left(p_{2} \partial_{q_{1}} r+q_{2} p_{2} \partial_{q_{1}} \partial_{q_{2}} r-\left(2 r \partial_{q_{2}} r+\partial_{q_{2}} r+q_{2} \partial_{q_{2}}^{2} r\right) p_{1}\right) \frac{\partial u}{\partial p_{2}} \\
+\left(r^{2}+q_{2} \partial_{q_{2}} r+\left(2 r \partial_{p_{1}} r+q_{2} \partial_{q_{2}} \partial_{p_{1}} r\right) p_{1}-q_{2} p_{2} \partial_{p_{1}} \partial_{q_{1}} r\right) \frac{\partial u}{\partial q_{1}} \\
+\quad\left(-q_{2} \partial_{q_{1}} r-q_{2} p_{2} \partial_{q_{1}} \partial_{p_{2}} r+p_{1}\left(2 r \partial_{p_{2}} r+q_{2} \partial_{q_{2}} \partial_{p_{2}} r\right)\right) \frac{\partial u}{\partial q_{2}}=0 .
\end{gathered}
$$

We define

$$
v \equiv v\left(q_{1}, p_{1}, p_{2}\right):=u\left(q_{1}, 0, p_{1}, p_{2}\right)
$$


By setting $q_{2}=0$ in (3.1) and noting that $\partial_{q_{2}} r\left(q_{1}, 0\right) \equiv 0$ and $r\left(q_{1}, 0\right)=q_{1}^{2 \sigma}$ by $(2.1)$, we obtain

$$
2 \sigma p_{2} \frac{\partial v}{\partial p_{2}}-4 \sigma q_{1}^{2 \sigma} p_{1} \frac{\partial v}{\partial p_{1}}+q_{1}^{2 \sigma+1} \frac{\partial v}{\partial q_{1}}=0
$$

We expand $v$ into the power series of $p_{2}, v=\sum_{j=0}^{\infty} v_{j}\left(q_{1}, p_{1}\right) p_{2}^{j}$. Then we see that $v_{j}\left(q_{1}, p_{1}\right)(j=0,1, \ldots)$ satisfy

$$
2 \sigma j v_{j}-4 \sigma q_{1}^{2 \sigma} p_{1} \frac{\partial v_{j}}{\partial p_{1}}+q_{1}^{2 \sigma+1} \frac{\partial v_{j}}{\partial q_{1}}=0, \quad j=0,1,2, \ldots
$$

We want to show that $v_{j}=0$ if $j \neq 0$, and $v=\phi\left(p_{1} q_{1}^{4 \sigma}\right)$ for some analytic function $\phi(t)$ of one variable. Indeed, by substituting the expansion of $v_{j} v_{j}\left(q_{1}, p_{1}\right)=$ $\sum_{\nu=0}^{\infty} v_{j, \nu}\left(q_{1}\right) p_{1}^{\nu}$ into (3.4) we obtain

$$
2 \sigma j v_{j, \nu}-4 \sigma \nu q_{1}^{2 \sigma} v_{j, \nu}+q_{1}^{2 \sigma+1} \frac{\partial v_{j, \nu}}{\partial q_{1}}=0, \quad j=0,1,2, \ldots
$$

If we expand $v_{j, \nu}$ into the power series of $q_{1}$, then we can easily see that $v_{j, \nu} \equiv 0$ for all $\nu=0,1, \ldots$, if $j \neq 0$. Hence we have $v_{j}=0$ if $j \neq 0$. It follows that $v=v_{0}\left(q_{1}, p_{1}\right)$. Moreover, by (3.4) $v$ satisfies the equation

$$
-4 \sigma p_{1} \frac{\partial v}{\partial p_{1}}+q_{1} \frac{\partial v}{\partial q_{1}}=0
$$

If we substitute the expansion of $v$ into the equation, then, by simple computations, we easily see that $v=\phi\left(p_{1} q_{1}^{4 \sigma}\right)$ for some analytic function $\phi(t)$ of one variable. This proves the assertion.

It follows from $(2.2)$ that $v=v_{0}=\phi\left(p_{1} q_{1}^{4 \sigma}\right)=\phi\left(\left.\mathcal{H}\right|_{q_{2}=0}\right)$. We define

$$
g\left(q_{1}, q_{2}, p_{1}, p_{2}\right):=u\left(q_{1}, q_{2}, p_{1}, p_{2}\right)-\phi(\mathcal{H}) .
$$

By (3.2) and by recalling that $\mathcal{H}$ is a first integral we see that $g$ is an analytic solution of $(3.1)$ such that $g\left(q_{1}, 0, p_{1}, p_{2}\right) \equiv 0$. In order to prove Theorem 2.1 we shall show $g\left(q_{1}, q_{2}, p_{1}, p_{2}\right) \equiv 0$ in some neighborhood of the origin. First we will show that

$$
g\left(q_{1}, q_{2}, p_{1}, p_{2}\right)=\phi_{1}\left(p_{1} q_{1}^{4 \sigma}\right) p_{2} q_{2}+h_{2}\left(q_{1}, p_{1}, p_{2}\right) q_{2}^{2}+\tilde{h}_{3}\left(q_{1}, q_{2}, p_{1}, p_{2}\right) q_{2}^{3},
$$

for some analytic function $\phi_{1}$ of one variable and analytic functions $h_{2}$ and $\tilde{h}_{3}$. Because $g$ is analytic we have the expansion

$$
g\left(q_{1}, q_{2}, p_{1}, p_{2}\right)=g_{1}\left(q_{1}, p_{1}, p_{2}\right) q_{2}+h_{2}\left(q_{1}, p_{1}, p_{2}\right) q_{2}^{2}+\tilde{h}_{3}\left(q_{1}, q_{2}, p_{1}, p_{2}\right) q_{2}^{3} .
$$

We substitute (3.8) with $u=g$ into (3.1) and compare the coefficients of $q_{2}$. By (2.1) we have

$$
-4 \sigma q_{1}^{2 \sigma} p_{1} \frac{\partial g_{1}}{\partial p_{1}}+2 \sigma\left(p_{2} \frac{\partial g_{1}}{\partial p_{2}}-g_{1}\right)+q_{1}^{2 \sigma+1} \frac{\partial g_{1}}{\partial q_{1}}=0
$$


By substituting the expansion $g_{1}\left(q_{1}, p_{1}, p_{2}\right)=\sum_{m=0}^{\infty} g_{1, m}\left(q_{1}, p_{1}\right) p_{2}^{m}$ into (3.9) and by comparing the coefficients of $p_{2}^{m}$ we obtain

$$
-4 \sigma q_{1}^{2 \sigma} p_{1} \frac{\partial g_{1, m}}{\partial p_{1}}+2 \sigma(m-1) g_{1, m}+q_{1}^{2 \sigma+1} \frac{\partial g_{1, m}}{\partial q_{1}}=0 .
$$

If $m \neq 1$, then we obtain a similar equation as for $v_{j}$ in (3.4). Hence we have $g_{1, m}=0$ if $m \neq 1$. It follows that $g_{1}=g_{1,1} p_{2}$, and $g_{1,1}$ satisfies the equation $-4 \sigma p_{1} \frac{\partial g_{1,1}}{\partial p_{1}}+q_{1} \frac{\partial g_{1,1}}{\partial q_{1}}=$ 0 . By the same argument as in the above, we see that $g_{1}=g_{1,1} p_{2}=\phi_{1}\left(p_{1} q_{1}^{4 \sigma}\right) p_{2}$ for some analytic function $\phi_{1}$ of one variable. This proves the assertion.

Let us now suppose that

$$
\begin{aligned}
g\left(q_{1}, q_{2}, p_{1}, p_{2}\right) & =\phi_{n-1}\left(p_{1} q_{1}^{4 \sigma}\right) p_{2}^{n-1} q_{2}^{n-1} \\
& +h_{n}\left(q_{1}, p_{1}, p_{2}\right) q_{2}^{n}+\tilde{h}_{n+1}\left(q_{1}, q_{2}, p_{1}, p_{2}\right) q_{2}^{n+1},
\end{aligned}
$$

for some $n \geq 2$, some analytic function $\phi_{n-1}$ of one variable and analytic functions $h_{n}\left(q_{1}, p_{1}, p_{2}\right)$ and $\tilde{h}_{n+1}\left(q_{1}, q_{2}, p_{1}, p_{2}\right)$. Then we substitute (3.11) into (3.1) with $u=g$ and we compare the coefficients of $q_{2}^{n}$. By (2.1) we have

$$
\begin{aligned}
& 2 p_{2}^{n} \sigma(2 \sigma-1) q_{1}^{6 \sigma-2} \phi_{n-1}^{\prime}-4 a\left(q_{1}^{2 \sigma}\right)\left(q_{1}^{2 \sigma}+1\right)(n-1) p_{1} p_{2}^{n-2} \phi_{n-1} \\
- & 4 \sigma q_{1}^{4 \sigma-1} p_{1} \frac{\partial h_{n}}{\partial p_{1}}+2 \sigma q_{1}^{2 \sigma-1}\left(p_{2} \frac{\partial h_{n}}{\partial p_{2}}-n h_{n}\right)+q_{1}^{4 \sigma} \frac{\partial h_{n}}{\partial q_{1}}=0 .
\end{aligned}
$$

By substituting the expansion $h_{n}\left(q_{1}, p_{1}, p_{2}\right)=\sum_{m=0}^{\infty} h_{n, m}\left(q_{1}, p_{1}\right) p_{2}^{m}$ into (3.12) and by comparing the coefficients of $p_{2}^{n-2}$ we obtain

$$
\begin{aligned}
-4 \sigma q_{1}^{4 \sigma-1} p_{1} \frac{\partial h_{n, n-2}}{\partial p_{1}} & -4 a\left(q_{1}^{2 \sigma}\right)\left(q_{1}^{2 \sigma}+1\right)(n-1) p_{1} \phi_{n-1} \\
& -4 \sigma q_{1}^{2 \sigma-1} h_{n, n-2}+q_{1}^{4 \sigma} \frac{\partial h_{n, n-2}}{\partial q_{1}}=0
\end{aligned}
$$

We will show that

$$
h_{n, n-2}=0, \quad \phi_{n-1}=0 .
$$

If we can prove $\phi_{n-1}=0$, then it follows from (3.13) that $v:=h_{n, n-2}$ satisfies a similar equation as (3.4). Hence, by the same argument as for (3.4) we have $h_{n, n-2}=$ 0 . In order to show $\phi_{n-1}=0$ we insert the expansions

$$
\phi_{n-1}\left(p_{1} q_{1}^{4 \sigma}\right)=\sum_{k=0}^{\infty} \phi_{n-1, k} p_{1}^{k} q_{1}^{4 \sigma k}, \quad h_{n, n-2}\left(q_{1}, p_{1}\right)=\sum_{k=0}^{\infty} h_{n, n-2, k}\left(q_{1}\right) p_{1}^{k}
$$

into (3.13) and we compare the coefficients of $p_{1}^{k}$. Then we obtain, for $k \geq 0$

$$
\begin{aligned}
-4 \sigma q_{1}^{4 \sigma-1} k h_{n, n-2, k} & -4 \sigma q_{1}^{2 \sigma-1} h_{n, n-2, k}+q_{1}^{4 \sigma} \frac{\partial h_{n, n-2, k}}{\partial q_{1}} \\
& =4 a\left(q_{1}^{2 \sigma}\right)\left(q_{1}^{2 \sigma}+1\right)(n-1) \phi_{n-1, k-1} q_{1}^{4 \sigma(k-1)},
\end{aligned}
$$

where we set $\phi_{n-1,-1}=0$. If we set $q_{1}=0$ and $k=1$ in (3.16), then, by $a(0)=1$, we obtain $0=4(n-1) \phi_{n-1,0}$, which implies $\phi_{n-1,0}=0$. 
Suppose that $\phi_{n-1, k-1} \neq 0$ for some $k \geq 2$. We divide both sides of (3.16) by $q_{1}^{2 \sigma-1}$. Then the right-hand side of (3.16) is divisible by $q_{1}^{N}, N=4 \sigma(k-1)+1-2 \sigma \geq$ $2 \sigma+1$. Because the operator $-4 \sigma k q_{1}^{2 \sigma}+q_{1}^{2 \sigma+1}\left(d / d q_{1}\right)$ in the left-hand side of the equation increases the power of $q_{1}$, it follows that $h_{n, n-2, k}$ is divisible by $q_{1}^{N}$. We set $h_{n, n-2, k}\left(q_{1}\right)=q_{1}^{N} W\left(q_{1}\right)$. Then we have $q_{1}\left(d / d q_{1}\right) h_{n, n-2, k}=q_{1}^{N}\left(N+q_{1}\left(d / d q_{1}\right)\right) W$. It follows from (3.16) that $W$ satisfies

$$
\begin{aligned}
(N-4 \sigma k) q_{1}^{2 \sigma} W-4 \sigma W & +q_{1}^{2 \sigma+1} \frac{d W}{d q_{1}} \\
& =4 a\left(q_{1}^{2 \sigma}\right)(n-1) \phi_{n-1, k-1}\left(q_{1}^{2 \sigma}+1\right) .
\end{aligned}
$$

We set $W=\sum_{j=0}^{2 \sigma-1} q_{1}^{j} W_{j}\left(q_{1}^{2 \sigma}\right)$. Because the right-hand side of (3.17) is a function of $q_{1}^{2 \sigma}, W_{j}(1 \leq j<2 \sigma)$ satisfy

$$
q_{1}^{2 \sigma}(N-4 \sigma k+j) W_{j}-4 \sigma W_{j}+q_{1}^{2 \sigma+1} \frac{d W_{j}}{d q_{1}}=0 .
$$

By a similar argument as for (3.4) we have $W_{j}=0$ for $1 \leq j<2 \sigma$. Hence we have $W\left(q_{1}\right)=W_{0}\left(q_{1}^{2 \sigma}\right)=: V(t)\left(t=q_{1}^{2 \sigma}\right)$. Because $q_{1}\left(d / d q_{1}\right) V=2 \sigma t(d / d t) V$, it follows from (3.17) that

$$
(1-6 \sigma) t V \quad-4 \sigma V+2 \sigma t^{2} \frac{d V}{d t}=4 a(t)(n-1)(t+1) \phi_{n-1, k-1}
$$

If we set $U:=V /\left(2(n-1) \phi_{n-1, k-1}\right)$, then $U$ is an analytic solution of (2.4). This contradicts to the assumption of the theorem, because we assume that (M) is not verified. Hence we have $\phi_{n-1, k-1}=0$. Because $k$ is arbitrary we have $\phi_{n-1}=0$.

Next we set $\phi_{n-1}=0$ in (3.12) and consider the coefficients of $p_{2}^{m}(m \neq n)$. Then we see that $h_{n, m}$ satisfies a similar equation as for (3.4). Hence we have $h_{n, m}=0$ if $n \neq m$, and $h_{n, n}=\phi_{n}\left(p_{1} q_{1}^{4 \sigma}\right)$ for some analytic function $\phi_{n}$ of one variable. It follows that $h_{n}\left(q_{1}, p_{1}, p_{2}\right)=h_{n, n}\left(q_{1}, p_{1}\right) p_{2}^{n}=\phi_{n}\left(p_{1} q_{1}^{4 \sigma}\right) p_{2}^{n}$. Hence we have (3.11) with $n$ replaced by $n+1$. By induction we obtain (3.11) for an arbitrary integer $n \geq 2$.

It follows from (3.11) with $n$ replaced by $n+2$ that, for every $n \geq 0$ we have $\partial_{q_{2}}^{n} g\left(q_{1}, 0, p_{1}, p_{2}\right) \equiv 0$, where $\left(q_{1}, p_{1}, p_{2}\right)$ is in some neighborhood of the origin which may depend on $n$. On the other hand $\partial_{q_{2}}^{n} g\left(q_{1}, 0, p_{1}, p_{2}\right)$ is analytic in some neighborhood of the origin independent of $n$. By analytic continuation, we have $\partial_{q_{2}}^{n} g\left(q_{1}, 0, p_{1}, p_{2}\right)$ $\equiv 0$ in some neighborhood of the origin independent of $n$. By the partial Taylor expansion $g=\sum_{n} \partial_{q_{2}}^{n} g\left(q_{1}, 0, p_{1}, p_{2}\right) q_{2}^{n} / n$ !, we have $g=0$.

\section{TRANSSERIES EXPANSION OF FIRST INTEGRAL}

In this section, we shall construct a first integral of (2.3) as a transseries. In order to introduce such a series we consider the terms in (3.1) which preserve the order of $q_{2}$

$$
\mathcal{L} u:=q_{1}^{2 \sigma-1}\left(2 \sigma\left(p_{2} \frac{\partial u}{\partial p_{2}}-q_{2} \frac{\partial u}{\partial q_{2}}\right)+q_{1}^{2 \sigma}\left(q_{1} \frac{\partial u}{\partial q_{1}}-4 \sigma p_{1} \frac{\partial u}{\partial p_{1}}\right)\right)
$$


We note that we can write (3.1) in the form

$$
\mathcal{L} u+R u=0, \quad R u:=\{\mathcal{H}, u\}-\mathcal{L} u .
$$

In order to construct an inverse of $\mathcal{L}$ we consider the Lagrange-Charpit system corresponding to $\mathcal{L}$

$$
\frac{d q_{1}}{q_{1}^{4 \sigma}}=\frac{d q_{2}}{-2 \sigma q_{1}^{2 \sigma-1} q_{2}}=\frac{d p_{1}}{-4 \sigma q_{1}^{4 \sigma-1} p_{1}}=\frac{d p_{2}}{2 \sigma q_{1}^{2 \sigma-1} p_{2}} .
$$

We integrate (4.3) by taking $q_{1}$ as an independent variable. By simple computations we can easily see that the solution of (4.3) is given by

$$
q_{2}=q_{2}^{0} \exp \left(q_{1}^{-2 \sigma}\right), p_{2}=p_{2}^{0} \exp \left(-q_{1}^{-2 \sigma}\right), p_{1}=p_{1}^{0} q_{1}^{-4 \sigma},
$$

where $q_{2}^{0}, p_{2}^{0}$ and $p_{1}^{0}$ are certain constants.

We note that the solution of the homogeneous equation $\mathcal{L} v=0$ is given by

$$
v=\phi\left(p_{1} q_{1}^{4 \sigma}, p_{2} \exp \left(q_{1}^{-2 \sigma}\right), q_{2} \exp \left(-q_{1}^{-2 \sigma}\right)\right),
$$

with $\phi\left(p_{1}^{0}, p_{2}^{0}, q_{2}^{0}\right)$ being an arbitrary function of $p_{1}^{0}, p_{2}^{0}$ and $q_{2}^{0}$. We then construct a solution $u$ of (4.2) in the form

$$
u=\sum_{j=0}^{\infty} u_{j}\left(q_{1}, p_{1} q_{1}^{4 \sigma}, p_{2} \exp \left(q_{1}^{-2 \sigma}\right)\right)\left(\exp \left(-q_{1}^{-2 \sigma}\right) q_{2}\right)^{j}
$$

where $u_{0}\left(q_{1}, p_{1} q_{1}^{4 \sigma}, p_{2} \exp \left(q_{1}^{-2 \sigma}\right)\right) \equiv u_{0}\left(p_{1} q_{1}^{4 \sigma}, p_{2} \exp \left(q_{1}^{-2 \sigma}\right)\right)$. We call (4.6) the transseries solution of (4.2). Then we have

Proposition 4.1. Let $u_{0}\left(p_{1}^{0}, p_{2}^{0}\right)$ be a given analytic function of $p_{1}^{0}$ and $p_{2}^{0}$ such that $\partial u_{0} / \partial p_{2}^{0} \neq 0$. Then (2.3) is formally Liouville-integrable in the sense that (4.6) is a formal integral of (2.3) which is functionally independent of $\mathcal{H}$.

Proof. We note that $R$ in (4.2) has analytic coefficients and $R$ raises the power of $q_{2}$ at least by one. On the other hand we have

$$
\mathcal{L}\left(u_{j}\left(\exp \left(-q_{1}^{-2 \sigma}\right) q_{2}\right)^{j}\right)=\left(\mathcal{L} u_{j}\right)\left(\exp \left(-q_{1}^{-2 \sigma}\right) q_{2}\right)^{j} .
$$

Hence, if we substitute (4.6) into (4.2) and compare the coefficients of $q_{2}^{j}$ of both sides, then we have

$$
\mathcal{L} u_{j}=\left(\text { linear functions of } u_{k} \text { and their derivatives }(k<j), \quad j=1,2, \ldots\right.
$$

We note that the right-hand side is a known quantity if we determine $u_{j}$ recursively.

We will solve $\mathcal{L} v=f$, where

$$
v=v\left(q_{1}, p_{1} q_{1}^{4 \sigma}, p_{2} \exp \left(q_{1}^{-2 \sigma}\right)\right) .
$$

By making the change of variables $\left(q_{1}, p_{1}, p_{2}\right) \mapsto\left(q_{1}, p_{1}^{0}, p_{2}^{0}\right)$ given by $(4.4)$, the equation $\mathcal{L} v=f\left(q_{1}, p_{1}, p_{2}\right)$ is written in the form

$$
q_{1}^{4 \sigma}\left(\partial v / \partial q_{1}\right)=g\left(q_{1}, p_{1}^{0}, p_{2}^{0}\right)
$$


where

$$
g \equiv g\left(q_{1}, p_{1}^{0}, p_{2}^{0}\right)=f\left(q_{1}, p_{1}^{0} q_{1}^{-4 \sigma}, p_{2}^{0} \exp \left(-q_{1}^{-2 \sigma}\right)\right) .
$$

Hence the solution of (4.9) is given by

$$
v=\int_{a_{0}}^{q_{1}} s^{-4 \sigma} g\left(s, p_{1}^{0}, p_{2}^{0}\right) d s,
$$

where $a_{0}$ is an arbitrary complex constant. If we go back to the original variables $q_{1}$, $p_{1}$ and $p_{2}$, then we obtain a solution of $\mathcal{L} v=f$. Therefore we have a solution $u$ of (4.2) given by (4.6).

Finally, we will show that $u$ converges, then $u$ is an integral of (2.3) functionally independent of $\mathcal{H}$. Hence our Hamiltonian system is formally Liouville-integrable. Indeed, if this is not the case, then we have $u=\phi(\mathcal{H})$ for some smooth function $\phi$ of one variable. If we set $q_{2}=0$, then we obtain

$$
u_{0}\left(p_{1} q_{1}^{4 \sigma}, p_{2} \exp \left(q_{1}^{-2 \sigma}\right)\right)=\left.\phi(\mathcal{H})\right|_{q_{2}=0}=\phi\left(p_{1} q_{1}^{4 \sigma}\right) .
$$

This is a contradiction to the assumption that $\partial u_{0} / \partial p_{2}^{0} \neq 0$. This ends the proof.

\section{Convergence of transseries}

$$
\mathcal{H}=-2 \sigma q_{1}^{2 \sigma-1} q_{2} p_{2}\left(1+q_{2}^{2} a^{\prime}\right)+p_{1}\left(\left(q_{1}^{2 \sigma}+a q_{2}^{2}\right)^{2}+2 a q_{2}^{2}\right)
$$

where we assume $a(0)=1$ for the sake of simplicity. We study the convergence of transseries solutions (4.6), where we set $a_{0}=0$ in (4.10). Note that $u_{j}\left(0, p_{1}^{0}, p_{2}^{0}\right)$ identically vanishes for any $j \geq 1$.

Clearly, the integral $u$ is the solution of (4.2), where $\mathcal{L}$ and $R$ are given, respectively, by (4.1) and

$$
\begin{aligned}
R u & =\left(\tilde{\alpha} p_{1}+\tilde{\beta} p_{2}\right) \frac{\partial u}{\partial p_{1}}+\left(\tilde{\gamma} p_{2}+\tilde{\delta} p_{1}\right) \frac{\partial u}{\partial p_{2}} \\
& +E\left(q_{1}, q_{2}\right) \frac{\partial u}{\partial q_{1}}-2 \sigma q_{1}^{2 \sigma-1} a^{\prime} q_{2}^{3} \frac{\partial u}{\partial q_{2}}
\end{aligned}
$$

where

$$
\begin{aligned}
E & \equiv E\left(q_{1}, q_{2}\right):=a q_{2}^{2}\left(a q_{2}^{2}+2 q_{1}^{2 \sigma}+2\right), \\
\tilde{\alpha} & :=-4 \sigma q_{1}^{2 \sigma-1} q_{2}^{2}\left(a+a^{\prime}+a^{\prime} q_{1}^{2 \sigma}+a a^{\prime} q_{2}^{2}\right), \\
\tilde{\beta} & :=2 \sigma(2 \sigma-1) q_{2} q_{1}^{2 \sigma-2}\left(1+a^{\prime} q_{2}^{2}\right)+4 \sigma^{2} q_{1}^{4 \sigma-2} a^{\prime \prime} q_{2}^{3}, \\
\tilde{\gamma} & :=6 \sigma q_{1}^{2 \sigma-1} a^{\prime} q_{2}^{2}, \quad \tilde{\delta}:=-4 q_{2} a\left(q_{1}^{2 \sigma}+a q_{2}^{2}+1\right) .
\end{aligned}
$$

Let $\varepsilon_{0}$ be a small positive constant. Then we define

$$
S_{0}:=\left\{q_{1} \in \mathbb{C} ;\left|q_{1}\right|<\varepsilon_{0}\right\} \cap\left\{q_{1} \in \mathbb{C} ; \operatorname{Re} q_{1}^{2 \sigma}<0\right\},
$$

where $\operatorname{Re} q_{1}^{2 \sigma}$ denotes the real part of $q_{1}^{2 \sigma}$. Then we have 
THEOREM 5.1. Let $u_{0}\left(p_{1}^{0}, p_{2}^{0}\right)$ be an analytic function of $p_{1}^{0}$ and $p_{2}^{0}$ such that $\partial u_{0} / \partial p_{2}^{0} \neq$ 0 in some neighborhood of the origin. Then there exist a $\delta>0$, an $\varepsilon_{0}>0$, neighborhoods $V_{1}, V_{2}$ of the origin in $\mathbb{C}$ such that $u$ in (4.6) converges in the domain

$$
\left\{\left(q_{1}, q_{2}, p_{1}, p_{2}\right) ; q_{1} \in S_{0}, p_{1} \in V_{1}, p_{2} \in V_{2},\left|\exp \left(-q_{1}^{-2 \sigma}\right) q_{2}\right|<\delta\right\} .
$$

Proof. Let $\mathcal{L}$ be given by (4.1). The Lagrange-Charpit system corresponding to $\mathcal{L}+R$ is given by

$$
\frac{d q_{1}}{q_{1}^{4 \sigma}+E}=\frac{d q_{2}}{-2 \sigma q_{1}^{2 \sigma-1} q_{2} T}=\frac{d p_{1}}{\left(\tilde{\alpha}-4 \sigma q_{1}^{4 \sigma-1}\right) p_{1}+\tilde{\beta} p_{2}}=\frac{d p_{2}}{\left(2 \sigma q_{1}^{2 \sigma-1}+\tilde{\gamma}\right) p_{2}+\tilde{\delta} p_{1}}
$$

where we set

$$
T \equiv T\left(q_{1}, q_{2}\right):=1+a^{\prime} q_{2}^{2} .
$$

We integrate (5.8) by taking $q_{1} \in S_{0}$ as an independent variable. We want to show that the solutions are perturbations of the solutions (4.4) in $S_{0}$. Namely, we will prove

$$
\begin{aligned}
& q_{2}=q_{2}^{0} \exp \left(q_{1}^{-2 \sigma}\right) \exp \left(q_{1} Q_{2}\left(q_{1}, q_{2}^{0}\right)\right) \\
& p_{1}=q_{1}^{-4 \sigma}\left(p_{1}^{0}\left(1+P_{1}\left(q_{1}, q_{2}^{0}\right)\right)+p_{2}^{0} \tilde{P}_{1}\left(q_{1}, q_{2}^{0}\right)\right) \\
& p_{2}=\exp \left(-q_{1}^{-2 \sigma}\right)\left(p_{1}^{0} P_{2}\left(q_{1}, q_{2}^{0}\right)+p_{2}^{0}\left(1+\tilde{P}_{2}\left(q_{1}, q_{2}^{0}\right)\right)\right)
\end{aligned}
$$

for some functions $Q_{2} \equiv Q_{2}\left(q_{1}, q_{2}^{0}\right), P_{j} \equiv P_{j}\left(q_{1}, q_{2}^{0}\right)$ and $\tilde{P}_{j} \equiv \tilde{P}_{j}\left(q_{1}, q_{2}^{0}\right)(j=1,2)$, which are holomorphic and bounded when $q_{1} \in S_{0}$ and $q_{2}^{0}$ in some neighborhood of $q_{2}^{0}=0$. Here $p_{1}^{0}$ and $p_{2}^{0}$ are arbitrary constants.

In order to verify these properties we first consider the following equation

$$
\frac{d q_{2}}{d q_{1}}=\frac{-2 \sigma q_{2} q_{1}^{2 \sigma-1}\left(1+a^{\prime} q_{2}^{2}\right)}{q_{1}^{4 \sigma}+E}=\frac{-2 \sigma q_{2}}{q_{1}^{2 \sigma+1}}\left(1+\frac{a^{\prime} q_{2}^{2}-q_{1}^{-4 \sigma} E}{1+q_{1}^{-4 \sigma} E}\right) .
$$

Clearly, $q_{2}=0$ is a solution of (5.13). We assume $q_{2} \not \equiv 0$. We note that $v:=$ $q_{2}^{0} \exp \left(q_{1}^{-2 \sigma}\right)$ satisfies the equation $d v / d q_{1}=-2 \sigma v q_{1}^{-2 \sigma-1}$. We set $U:=q_{1} Q_{2}$, and we substitute (5.10) into (5.13). Then we have

$$
\frac{d U}{d q_{1}}=-\frac{2 \sigma\left(a^{\prime} q_{2}^{2}-q_{1}^{-4 \sigma} E\right)}{q_{1}^{2 \sigma+1}\left(1+q_{1}^{-4 \sigma} E\right)}=: f\left(q_{1}, U\right)
$$

where $q_{2}=q_{2}^{0} \exp \left(q_{1}^{-2 \sigma}\right) e^{U}$. Because $\operatorname{Re} q_{1}^{2 \sigma}<0$ in $S_{0}$, we see that $f\left(q_{1}, U\right)$ is holomorphic when $\left(q_{1}, U\right) \in S_{0} \times \Omega$, and continuous up to its closure, where $\Omega$ is a neighborhood of the origin. Moreover, its maximal norm when $\left(q_{1}, U\right) \in S_{0} \times \Omega$ can be made arbitrarily small if we shrink $S_{0}$ sufficiently small.

We will solve (5.14) in $S_{0}$. If we replace $U$ with $U+c$ for a constant $c$ we see from (5.10) that $q_{2}^{0}$ is replaced by $e^{c} q_{2}^{0}$. Hence we may assume that $U$ vanishes at $q_{1}=0$. We will look for the solution $U$ as the solution of the following equation

$$
U=\int_{0}^{q_{1}} f(s, U) d s
$$


where the integral is taken along the straight line in $S_{0}$ which connects 0 and $q_{1}$. We note that we can make $\left|f\left(q_{1}, U\right)\right|$ arbitrarily small if we take $\varepsilon_{0}$ in $S_{0}$ sufficiently small and $U$ is bounded. We can easily show that the right-hand side operator of (5.15) is a contraction mapping on a small ball in the set of functions holomorphic in $S_{0}$ and continuous on its closure, and $U(0)=0$. Hence we have a holomorphic solution $U$ in $S_{0}$ of (5.15). If we set $Q_{2}:=q_{1}^{-1} U$ we obtain the desired solution. The analyticity of $U$ with respect to $q_{2}^{0}$ is easy to verify because of the definition of $f$.

Next we study the equations for $p_{1}$ and $p_{2}$. It follows from (5.8) that

$$
\frac{d p_{1}}{d q_{1}}=\alpha p_{1}+\beta p_{2}, \quad \frac{d p_{2}}{d q_{1}}=\gamma p_{2}+\delta p_{1}
$$

where

$$
\alpha=\frac{\tilde{\alpha}-4 \sigma q_{1}^{4 \sigma-1}}{q_{1}^{4 \sigma}+E}, \beta=\frac{\tilde{\beta}}{q_{1}^{4 \sigma}+E}, \gamma=\frac{2 \sigma q_{1}^{2 \sigma-1}+\tilde{\gamma}}{q_{1}^{4 \sigma}+E}, \delta=\frac{\tilde{\delta}}{q_{1}^{4 \sigma}+E} .
$$

We will construct the solution of (5.16) in the following form

$$
p_{\nu}=\sum_{j=0}^{\infty} p_{\nu}^{(j)}, \quad \nu=1,2
$$

where

$$
\frac{d p_{1}^{(0)}}{d q_{1}}=\alpha p_{1}^{(0)}, \quad \frac{d p_{2}^{(0)}}{d q_{1}}=\gamma p_{2}^{(0)}
$$

and $p_{\nu}^{(j)}(\nu=1,2 ; j=1,2, \ldots)$ are determined by

$$
\frac{d p_{1}^{(j)}}{d q_{1}}=\alpha p_{1}^{(j)}+\beta p_{2}^{(j-1)}, \quad \frac{d p_{2}^{(j)}}{d q_{1}}=\gamma p_{2}^{(j)}+\delta p_{1}^{(j-1)} .
$$

First we solve (5.19). By the definition of $\gamma$ in (5.17) we have the expression $\gamma=2 \sigma q_{1}^{-2 \sigma-1}+\gamma_{0}$, where $\gamma_{0}$ is a bounded holomorphic function in $S_{0}$. By the change of an unknown function similar in the argument for (5.13) the solution $p_{2}^{(0)}$ of (5.19) has the following expression

$$
p_{2}^{(0)}=p_{2}^{0} \exp \left(-q_{1}^{-2 \sigma}\right)\left(1+\tilde{P}_{2}^{(0)}\left(q_{1}, q_{2}^{0}\right)\right)
$$

for some $\tilde{P}_{2}^{(0)}$ which is bounded and holomorphic in $q_{1} \in S_{0}$ and $q_{2}^{0}$ in some neighborhood of the origin, where $p_{2}^{0}$ is an arbitrary constant. Similarly, noting that $\alpha=-4 \sigma q_{1}^{-1}+\alpha_{0}$ for some bounded holomorphic function $\alpha_{0}$ in $S_{0}$ we see that the solution $p_{1}^{(0)}$ of (5.19) has the following expression

$$
p_{1}^{(0)}=p_{1}^{0} q_{1}^{-4 \sigma}\left(1+\tilde{P}_{1}^{(0)}\left(q_{1}, q_{2}^{0}\right)\right)
$$

for some $\tilde{P}_{1}^{(0)}$ which is bounded and holomorphic in $q_{1} \in S_{0}$ and $q_{2}^{0}$ in some neighborhood of the origin, where $p_{1}^{0}$ is an arbitrary constant. 
Now we assume that $p_{\nu}^{(k)}$ 's $(k=0,1, \ldots, j-1, \nu=1,2)$ are determined. We define $p_{\nu}^{(j)}$ by the following relations

$$
\begin{aligned}
p_{1}^{(j)} & =\int_{0}^{q_{1}} \exp \left(\int_{s}^{q_{1}} \alpha(t) d t\right) \beta(s) p_{2}^{(j-1)}(s) d s, \\
p_{2}^{(j)} & =\int_{0}^{q_{1}} \exp \left(\int_{s}^{q_{1}} \gamma(t) d t\right) \delta(s) p_{1}^{(j-1)}(s) d s .
\end{aligned}
$$

Let $\mu>4 \sigma-1$ be a given integer. Then we will show the following expressions for $p_{\nu}^{(j)}$

$$
p_{1}^{(j)}= \begin{cases}p_{1}^{0} q_{1}^{-4 \sigma+(\mu-2 \sigma) k} P_{1}^{(j)}\left(q_{1}, q_{2}^{0}\right) & (j=2 k, k=0,1,2, \ldots) \\ p_{2}^{0} q_{1}^{-2 \sigma-1+(\mu-2 \sigma) k} P_{1}^{(j)}\left(q_{1}, q_{2}^{0}\right) & (j=2 k+1, k=0,1,2, \ldots)\end{cases}
$$

$$
p_{2}^{(j)}= \begin{cases}p_{2}^{0} \exp \left(-q_{1}^{-2 \sigma}\right) q_{1}^{(\mu-2 \sigma) k} P_{2}^{(j)}\left(q_{1}, q_{2}^{0}\right) & (j=2 k, k=0,1, \ldots) \\ p_{1}^{0} \exp \left(-q_{1}^{-2 \sigma}\right) q_{1}^{-2 \sigma+1+(\mu-2 \sigma)(k+1)} P_{2}^{(j)}\left(q_{1}, q_{2}^{0}\right) & (j=2 k+1, k=0,1, \ldots)\end{cases}
$$

where $P_{1}^{(j)}$ and $P_{2}^{(j)}$ are bounded holomorphic functions of $q_{1} \in S_{0}$ and $q_{2}^{0}$ in some neighborhood of the origin such that, for some constants $C_{0}>0$ and $C_{1}>0$ and the neighborhood of the origin $U$ we have

$$
\sup _{q_{1} \in S_{0}, q_{2}^{0} \in U}\left|P_{1}^{(j)}\left(q_{1}, q_{2}^{0}\right)\right| \leq C_{0} C_{1}^{j}, \quad \sup _{q_{1} \in S_{0}, q_{2}^{0} \in U}\left|P_{2}^{(j)}\left(q_{1}, q_{2}^{0}\right)\right| \leq C_{0} C_{1}^{j}, \quad j=0,1, \ldots
$$

We have already proved (5.25) and (5.26) for $j=0$. Let $j=1$. Since we have $\alpha(t)=-4 \sigma t^{-1}+\alpha_{0}$ for some bounded holomorphic function $\alpha_{0}$ in $S_{0}$, we have

$$
\exp \left(\int_{s}^{q_{1}} \alpha d t\right)=s^{4 \sigma} q_{1}^{-4 \sigma}\left(1+O\left(s, q_{1}\right)\right)
$$

where the term $O\left(s, q_{1}\right)$ represents a function holomorphic and bounded in $S_{0}$. By the definition of $\beta(s)$ and (5.10) we have

$$
\beta(s)=q_{2}^{0} 2 \sigma(2 \sigma-1) \exp \left(s^{-2 \sigma}\right) s^{-2 \sigma-2}(1+O(s)),
$$

where the term $O(s)$ represents the function holomorphic and bounded in $S_{0}$. Hence we have

$$
\begin{aligned}
p_{1}^{(1)}\left(q_{1}\right) & =\int_{0}^{q_{1}} s^{4 \sigma} q_{1}^{-4 \sigma} q_{2}^{0} 2 \sigma(2 \sigma-1) s^{-2 \sigma-2} p_{2}^{0}\left(1+O\left(s, q_{1}\right)\right) d s \\
& =q_{1}^{-4 \sigma} p_{2}^{0} q_{2}^{0} 2 \sigma(2 \sigma-1) \int_{0}^{q_{1}} s^{2 \sigma-2}\left(1+O\left(s, q_{1}\right)\right) d s .
\end{aligned}
$$

The right-hand side of (5.29) can be written in the form $p_{2}^{0} q_{1}^{-2 \sigma-1} P_{2}^{(1)}\left(q_{1}, q_{2}^{0}\right)$ for some bounded holomorphic function $P_{2}^{(1)}\left(q_{1}, q_{2}^{0}\right)$ in $q_{1} \in S_{0}$ and $q_{2}^{0}$ in some neighborhood of the origin. This proves (5.25) for $j=1$. 
In order to estimate $p_{2}^{(1)}$ we first note that $\gamma=2 \sigma q_{1}^{-2 \sigma-1}\left(1+O\left(q_{1}\right)\right)$. It follows that

$$
\exp \left(\int_{s}^{q_{1}} \gamma\right)=\exp \left(-q_{1}^{-2 \sigma}+s^{-2 \sigma}+O\left(q_{1}, s\right)\right) .
$$

On the other hand we have

$$
\delta(s)=\frac{-4 a q_{2}\left(s^{2 \sigma}+a q_{2}^{2}+1\right)}{s^{4 \sigma}+E}=-4 a q_{2} s^{-2 \sigma}(1+O(s)), \quad q \in S_{0} .
$$

By (5.10) we have $a q_{2} s^{-2 \sigma-\mu}=O(s)$ in $S_{0}$, it follows that $\delta(s)=s^{\mu} O(s)$, where $\mu>4 \sigma-1$ is a given integer. In terms of the estimate of $p_{1}^{(0)}$ and (5.24) we have

$$
p_{2}^{(1)}=p_{1}^{0} \int_{0}^{q_{1}} \exp \left(-q_{1}^{-2 \sigma}+s^{-2 \sigma}\right) s^{\mu-4 \sigma} O\left(q_{1}, s\right) d s=p_{1}^{0} \exp \left(-q_{1}^{-2 \sigma}\right) q_{1}^{\mu+1-4 \sigma} O\left(q_{1}\right),
$$

where $O\left(q_{1}\right)$ is a bounded holomorphic function in $S_{0}$. This proves (5.25) and (5.26) for $j=0,1$.

Let us now assume that (5.25) and (5.26) hold up to some $k \geq 0$. We first show (5.25) for $j=2(k+1)$. By (5.23) with $j=2 k+2$, (5.26) with $j=2 k+1$ and the same argument as in the estimate of $p_{1}^{(1)}$ we obtain $(5.25)$ for $j=2(k+1)$. Next we show (5.26) for $j=2(k+1)$. By (5.24) with $j=2(k+1)$, (5.25) with $j=2 k+1$ and the same argument as in the estimate of $p_{2}^{(1)}$ we obtain $(5.26)$ with $j=2(k+1)$. Next we show (5.25) with $j=2(k+1)+1$. By (5.23) with $j=2 k+3$, (5.26) with $j=2(k+1)$ and the same argument as for the estimate of $p_{1}^{(1)}$ we obtain (5.25) with $j=2 k+3$. To show (5.26) with $j=2 k+3$ we use (5.24) with $j=2 k+3$, (5.25) with $j=2 k+2$ and the same argument as for the estimate of $p_{2}^{(1)}$. Hence we have proved (5.25) and (5.26). In order to show the estimate (5.27) we note that $p_{\nu}^{(j)}$ in (5.23) and (5.24) are determined by the integral of the previous ones multiplied by some bounded holomorphic functions in $S_{0}$. Hence we can easily show the desired estimates. It follows from (5.25) and (5.26) that the series (5.18) converges in $q_{1} \in S_{0}$ and $q_{2}^{0}$ in some neighborhood of the origin. Moreover we have the representations (5.11) and (5.12).

Now we construct the solution $u$ of $\{\mathcal{H}, u\}=0$ in the form (4.6) where $q_{2}^{0}, p_{1}^{0}$ and $p_{2}^{0}$ are given by $(5.10),(5.11)$ and (5.12). For the sake of simplicity we set $\tilde{\mathcal{L}}:=\mathcal{L}+R$. We regard $q_{2}^{0}$ as the function of $q_{1}$ and $q_{2}$ through the relation (5.10) in view of the implicit function theorem. In terms of (5.8) and the implicit function theorem we can easily see that $\tilde{\mathcal{L}} q_{2}^{0}=0$.

Next we prove that $\tilde{\mathcal{L}} p_{\nu}^{0}=0(\nu=1,2)$. Indeed, by (5.11) and (5.12) we can determine $p_{\nu}^{0}$ as a linear function of $p_{1}$ and $p_{2}$, which is also holomorphic in $q_{1} \in S_{0}$. By applying $\tilde{\mathcal{L}}$ to both sides of (5.11), we see that the left-hand side is equal to $\left(q_{1}^{4 \sigma}+E\right)\left(\alpha p_{1}+\beta p_{2}\right)$. On the other hand the right-hand side can be written in

$$
q_{1}^{-4 \sigma}\left(\left(\tilde{\mathcal{L}} p_{1}^{0}\right)\left(1+P_{1}\right)+\left(\tilde{\mathcal{L}} p_{2}^{0}\right) \tilde{P}_{1}\right)+\cdots
$$

where the dots denotes the terms which does not contain the differentiations of $p_{\nu}^{0}$. Because $\tilde{\mathcal{L}} q_{2}^{0}=0$, if the differentiations of $\tilde{\mathcal{L}}$ are applied to $q_{2}^{0}$, then it vanishes. Hence, 
in the dotted terms one may consider the differentiations with respect to $q_{1}$. In view of the definition of (5.8) one can show that the dotted term is then equal to the left-hand side. Therefore we obtain the following

$$
q_{1}^{-4 \sigma}\left(\left(\tilde{\mathcal{L}} p_{1}^{0}\right)\left(1+P_{1}\right)+\left(\tilde{\mathcal{L}} p_{2}^{0}\right) \tilde{P}_{1}\right)=0
$$

We similarly obtain

$$
\exp \left(-q_{1}^{-2 \sigma}\right)\left(\left(\tilde{\mathcal{L}} p_{1}^{0}\right) P_{2}+\left(\tilde{\mathcal{L}} p_{2}^{0}\right)\left(1+\tilde{P}_{2}\right)\right)=0 .
$$

It follows that $\tilde{\mathcal{L}} p_{\nu}^{0}=0(\nu=1,2)$.

By (5.8) we have $\tilde{\mathcal{L}}\left(u_{j}\left(q_{1}, p_{1}^{0}, p_{2}^{0}\right)\left(q_{2}^{0}\right)^{j}\right)=\left(\tilde{\mathcal{L}} u_{j}\right)\left(q_{2}^{0}\right)^{j}$ and

$$
\begin{aligned}
& \tilde{\mathcal{L}} u_{j}=\left(q_{1}^{4 \sigma}+E\right) \partial_{1} u_{j} \\
E= & \left(q_{2}^{0}\right)^{2} \exp \left(2 q_{1} Q_{2}+2 q_{1}^{-2 \sigma}\right) a\left(\left(q_{2}^{0}\right)^{2} \exp \left(2 q_{1} Q_{2}+2 q_{1}^{-2 \sigma}\right) a+2 q_{1}^{2 \sigma}+2\right),
\end{aligned}
$$

where the differentiation $\partial_{1}$ means the one with respect to the first variable by considering $q_{1}, p_{1}^{0}, p_{2}^{0}$ and $q_{2}^{0}$ as the independent variables. Hence we can write $\tilde{\mathcal{L}}=0$ in the following form

$$
\left(q_{1}^{4 \sigma}+E\right) \partial_{1} u=0 .
$$

Because $E$ is an exponentially small when $q_{1} \rightarrow 0\left(q_{1} \in S_{0}\right)$, it follows that $q_{1}^{4 \sigma}+E$ does not vanish identically in $S_{0}$. Hence $\tilde{\mathcal{L}} u=0$ is equivalent to $\partial_{1} u=0$.

In order to determine $u$ we study the transformation among two cordinates of transseries. First we set

$$
\tilde{q}_{2}^{0}=q_{2} \exp \left(-q_{1}^{-2 \sigma}\right), \quad \tilde{p}_{1}^{0}=p_{1} q_{1}^{4 \sigma}, \quad \tilde{p}_{2}^{0}=p_{2} \exp \left(q_{1}^{-2 \sigma}\right) .
$$

Then it follows from (5.10), (5.11) and (5.12) that

$$
\begin{aligned}
& \tilde{q}_{2}^{0}=q_{2}^{0} \exp \left(q_{1} Q_{2}\right), \\
& \tilde{p}_{1}^{0}=p_{1}^{0}\left(1+P_{1}\right)+p_{2}^{0} \tilde{P}_{1}, \\
& \tilde{p}_{2}^{0}=p_{1}^{0} P_{2}+p_{2}^{0}\left(1+\tilde{P}_{2}\right) .
\end{aligned}
$$

By (5.39) and the implicit function theorem we can express $\tilde{q}_{2}^{0}$ as a holomorphic function of $q_{2}^{0}$ in some neighborhood of the origin, $q_{2}^{0}=0$ when $q_{1} \in S_{0}$. Conversely, $q_{2}^{0}$ can be expressed as the holomorphic function of $\tilde{q}_{2}^{0}$ in some neighborhood of the origin, $\tilde{q}_{2}^{0}=0$ when $q_{1} \in S_{0}$. We also recall that $Q_{2}, P_{\nu}, \tilde{P}_{\nu}$ are holomorphic functions of $q_{2}^{0}$ in some neighborhood of the origin, $q_{2}^{0}=0$.

We replace $p_{1}^{0}, p_{2}^{0}$ and $q_{2}^{0}$ in (4.6) with $\tilde{p}_{1}^{0}, \tilde{p}_{2}^{0}$ and $\tilde{q}_{2}^{0}$, respectively and we rewrite (4.6) in the following form

$$
u\left(q_{1}, \tilde{q}_{2}^{0}, \tilde{p}_{1}^{0}, \tilde{p}_{2}^{0}\right)=\sum_{j=0}^{\infty} \tilde{u}_{j}\left(q_{1}, \tilde{p}_{1}^{0}, \tilde{p}_{2}^{0}\right)\left(\tilde{q}_{2}^{0}\right)^{j},
$$

where $\tilde{u}_{0}$ is independent of $q_{1}$ and analytic in some neighborhood of the origin, $\tilde{p}_{1}^{0}=0$, $\tilde{p}_{2}^{0}=0$. We recall that $\tilde{u}_{j}\left(0, \tilde{p}_{1}^{0}, \tilde{p}_{2}^{0}\right) \equiv 0$ for every $j \geq 1$. We substitute the relations 
(5.39), (5.40) and (5.41) into (4.6). Then we see that the power series (4.6) is expressed as the power series of $q_{2}^{0}, p_{2}^{0}$ and $p_{1}^{0}$

$$
u\left(q_{1}, \tilde{q}_{2}^{0}, \tilde{p}_{1}^{0}, \tilde{p}_{2}^{0}\right)=u_{0}\left(p_{1}^{0}, p_{2}^{0}\right)+u_{1}\left(p_{1}^{0}, p_{2}^{0}\right) q_{2}^{0}+u_{2}\left(p_{1}^{0}, p_{2}^{0}\right)\left(q_{2}^{0}\right)^{2}+\cdots
$$

where we used the relations $\partial_{1} u_{j}=\partial_{q_{1}} u_{j}=0$ which follows from the fact that $\partial_{1} u=0$.

We first determine $u_{0}$. In view of the constructions of $P_{\nu}$ and $\tilde{P}_{\nu}(\nu=1,2)$ we can easily see that these functions vanish when $q_{2}^{0}=0$. Moreover, in view of the construction we can easily see that if we expand $P_{\nu}$ and $\tilde{P}_{\nu}(\nu=1,2)$ into the power series of $q_{2}^{0}$ the coefficients vanish when $q_{1} \rightarrow 0$. We substitute the relations (5.39) (5.41) into (5.42) and we look for terms in $\tilde{u}_{0}\left(\tilde{p_{1}^{0}}, \tilde{p_{2}^{0}}\right)$ which does not contain $q_{2}^{0}$. We can easily see that the term is given by $\tilde{u}_{0}\left(p_{1}^{0}, p_{2}^{0}\right)$. Hence we have $u_{0}\left(p_{1}^{0}, p_{2}^{0}\right)=\tilde{u}_{0}\left(p_{1}^{0}, p_{2}^{0}\right)$. This shows that $u_{0}\left(p_{1}^{0}, p_{2}^{0}\right)$ is analytic at $p_{1}^{0}=0, p_{2}^{0}=0$. Next we will show that $u_{1}\left(p_{1}^{0}, p_{2}^{0}\right)=0$. For this purpose we calculate the limit of the coefficients of $q_{2}^{0}$ in (5.42) when $q_{1} \rightarrow 0$, where we substitute (5.39) - (5.41) into (5.42). In view of the definitions of $P_{\nu}$ and $\tilde{P}_{\nu}(\nu=1,2)$ we can easily see that $\lim _{q_{1} \rightarrow 0} P_{\nu}=0$ and $\lim _{q_{1} \rightarrow 0} \tilde{P}_{\nu}=0$. Hence we have $\lim _{q_{1} \rightarrow 0} \tilde{p}_{1}^{0}=p_{1}^{0}$ and $\lim _{q_{1} \rightarrow 0} \tilde{p}_{2}^{0}=p_{2}^{0}$. Therefore, if we neglect terms which vanish when $q_{1} \rightarrow 0$, then we have

$$
u_{1}\left(p_{1}^{0}, p_{2}^{0}\right)=\lim _{q_{1} \rightarrow 0} \tilde{u}_{1}\left(q_{1}, \tilde{p}_{1}^{0}, \tilde{p}_{2}^{0}\right)=\tilde{u}_{1}\left(0, p_{1}^{0}, p_{2}^{0}\right) \equiv 0 .
$$

Similarly we can show that $u_{j}\left(p_{1}^{0}, p_{2}^{0}\right) \equiv 0$ for all $j \geq 1$. Therefore the right-hand side of (5.43) converges. If we return to the original variables the series (4.6) converges. This ends the proof of the theorem.

Remark. The proof of Theorem 5.1 also show that if we are given an analytic solution $u=u_{0}\left(p_{1}^{0}, p_{2}^{0}\right)$, then we obtain the solution (4.6). Indeed, by (5.39) - (5.41) we rewrite $u_{0}\left(p_{1}^{0}, p_{2}^{0}\right)$ as an analytic function of $q_{1}, \tilde{p}_{1}^{0}$ and $\tilde{p}_{2}^{0}$ in the following form

$$
\tilde{u}_{0}\left(q_{1}, \tilde{p}_{1}^{0}, \tilde{p}_{2}^{0}\right)+\tilde{u}_{1}\left(q_{1}, \tilde{p}_{1}^{0}, \tilde{p}_{2}^{0}\right) \tilde{q}_{2}^{0}+\cdots
$$

If we consider terms which does not contain $\tilde{q}_{2}^{0}$, then we obtain that $\tilde{u}_{0}\left(q_{1}, \tilde{p}_{1}^{0}, \tilde{p}_{2}^{0}\right)=$ $u_{0}\left(\tilde{p}_{1}^{0}, \tilde{p}_{2}^{0}\right)$. Similarly we can show that $\left.\tilde{u}_{j}\right|_{q_{1}=0}=0$ for all $j \geq 1$. Hence we obtain the solution (4.6).

Remark. So far we studied the solvability of $\mathcal{L} u+R u=0$ in a neighborhood of the point $\left(q_{1}, q_{2}, p_{1}, p_{2}\right)=(0,0,0,0)$. We will briefly mention the solvability of $\mathcal{L} u+R u=0$ at other points. As to the solvability at the points $\left(q_{1}, q_{2}, p_{1}, p_{2}\right)$ such that $q_{1} \neq 0, q_{2}=0$, we see that the term $q_{1}^{4 \sigma} \partial / \partial q_{1}$ appears in $\mathcal{L} u+R u=0$, which implies that $\mathcal{L} u+R u=0$ is a nonsingular vector field. Hence, by the standard existence theorem for the noncharacteristic Cauchy problem, $\mathcal{L} u+R u=0$ is solvable under a suitable initial condition on $u$.

By a similar argument, we see that at the point such that $q_{2} \neq 0, q_{1}=0, p_{1} \neq 0$, either the coefficient of $\partial u / \partial p_{2}$ or that of $\partial u / \partial q_{1}$ does not vanish because $a(0) \neq 0$. Hence the vector field $\mathcal{L}+R$ is nonsingular. At the point such that $q_{2} \neq 0, q_{1}=0$, $p_{1}=0$ we easily see that if $2+a q_{2}^{2} \neq 0$, then the coefficient of $\partial u / \partial q_{1}$ does not vanish. Hence $\mathcal{L}+R$ is nonsingular.

Finally, in a neighborhood of the point $\left(q_{1}, q_{2}, p_{1}, p_{2}\right)$ such that $q_{1}=0, q_{2}=\infty$, $\mathcal{L} u+R$ is nonsingular because $a^{2} \partial / \partial q_{1}$ does not vanish. In every case we can construct 
a solution by solving a noncharateristic Cauchy problem. Summimg up the above, if the condition $\left(q_{1}, q_{2}\right) \neq(0,0)$ holds, then $\mathcal{L} u+R u=0$ is solvable under an appropriate intial condition except for some special cases.

\section{Asymptotic properties of transseries}

In Theorem 5.1 we proved the solvability of $\mathcal{L} u+R u=0$ by using a transseries solution near $q_{2}=0$ in some narrow regin near the origin. We want to prove the solvability in a larger region by using the Borel resummation and the formal transseries solution constructed in $\S 4$. More precisely we will prove

TheOREM 6.1. Suppose that $a \equiv 1$ in the equation $(\mathcal{L}+R) v=0$. Moreover, assume that $v=\sum_{j=0}^{\infty} u_{j}\left(q_{1}, p_{1}^{0}, p_{2}^{0}\right)\left(q_{2}^{0}\right)^{j}$ be the solution of the equation $(\mathcal{L}+R) v=0$ constructed in $\S 4$ for a polynomial $u_{0} \equiv u_{0}\left(p_{1}^{0}, p_{2}^{0}\right)$ such that $\partial u_{0} / \partial p_{2}^{0} \neq 0$. Then, for every $T_{0}>0$ there exist $\nu_{0}>0, \varepsilon_{0}>0$ and $\varepsilon_{1}>0$ such that, for every $\nu \geq \nu_{0}$ the equation $(\mathcal{L}+R) u=0$ has a solution

$$
u=\sum_{j=0}^{\nu} u_{j}\left(q_{1}, p_{1}^{0}, p_{2}^{0}\right)\left(q_{2}^{0}\right)^{j}+U\left(q_{1}, p_{1}, p_{2}, q_{2}\right)
$$

such that $U\left(q_{1}, p_{1}, p_{2}, q_{2}\right)$ is holomorphic in the domain

$$
\begin{aligned}
& \left\{\left(q_{1}, q_{2}, p_{1}, p_{2}\right) ;\left|\arg q_{1}^{2 \sigma}-\pi\right|<\pi / 2+\varepsilon_{0},\left|q_{2}\right|<\varepsilon_{1}\left|q_{1}^{2 \sigma}\right|\right. \\
& \left.\left|p_{1}\right|<T_{0}\left|q_{1}^{4 \sigma} \exp \left(-q_{1}^{-2 \sigma}\right)\right|,\left|p_{2}\right|<T_{0}\left|q_{1}^{4 \sigma} \exp \left(-q_{1}^{-2 \sigma}\right)\right|\right\} .
\end{aligned}
$$

In order to prove Theorem 6.1 we prepare some notation. In terms of the condition $a \equiv 1 R$ is given by (5.2) with

$$
\begin{aligned}
E & \equiv E\left(q_{1}, q_{2}\right)=q_{2}^{2}\left(q_{2}^{2}+2 q_{1}^{2 \sigma}+2\right), \quad \tilde{\alpha}=-4 \sigma q_{1}^{2 \sigma-1} q_{2}^{2}, \\
\tilde{\beta} & =2 \sigma(2 \sigma-1) q_{2} q_{1}^{2 \sigma-2}, \quad \tilde{\gamma}=0, \quad \tilde{\delta}=-4 q_{2}\left(q_{1}^{2 \sigma}+q_{2}^{2}+1\right) .
\end{aligned}
$$

Now we make the change of variables

$$
t=q_{1}^{2 \sigma}, \quad s t=q_{2} .
$$

The Jacobian is nonsingular if $q_{1} \neq 0$. We can easily see that

$$
q_{1} \frac{\partial}{\partial q_{1}}=2 \sigma\left(t \frac{\partial}{\partial t}-s \frac{\partial}{\partial s}\right), \quad q_{2} \frac{\partial}{\partial q_{2}}=s \frac{\partial}{\partial s} .
$$

Therefore we have

$$
q_{1}^{-2 \sigma+1} \mathcal{L}=2 \sigma\left(p_{2} \frac{\partial}{\partial p_{2}}-s \frac{\partial}{\partial s}\right)+2 \sigma t\left(t \frac{\partial}{\partial t}-s \frac{\partial}{\partial s}-2 p_{1} \frac{\partial}{\partial p_{1}}\right) .
$$

In order to calculate $q_{1}^{-2 \sigma+1} R$ we note

$$
q_{1}^{-2 \sigma+1} E \frac{\partial}{\partial q_{1}}=2 \sigma s^{2} t\left(s^{2} t^{2}+2 t+2\right)\left(t \frac{\partial}{\partial t}-s \frac{\partial}{\partial s}\right) .
$$

Similarly we have $q_{1}^{-2 \sigma+1} \tilde{\alpha}=-4 \sigma s^{2} t^{2}, q_{1}^{-2 \sigma+1} \tilde{\beta}=2 \sigma(2 \sigma-1) s t^{1-1 /(2 \sigma)}$ and

$$
q_{1}^{-2 \sigma+1} \tilde{\delta}=-4 s\left(t^{1+1 /(2 \sigma)}+s^{2} t^{2+1 /(2 \sigma)}+t^{1 /(2 \sigma)}\right) .
$$


Hence we have

$$
\begin{aligned}
& q_{1}^{-2 \sigma+1} R=\left(-4 p_{1} \sigma s^{2} t^{2}+p_{2} 2 \sigma(2 \sigma-1) s t^{1-1 /(2 \sigma)}\right) \frac{\partial}{\partial p_{1}} \\
- & 4 p_{1} s\left(t^{1+1 /(2 \sigma)}+s^{2} t^{2+1 /(2 \sigma)}+t^{1 /(2 \sigma)}\right) \frac{\partial}{\partial p_{2}} \\
+ & 2 \sigma s^{2} t\left(s^{2} t^{2}+2 t+2\right)\left(t \frac{\partial}{\partial t}-s \frac{\partial}{\partial s}\right) .
\end{aligned}
$$

Therefore, by (6.6) and (6.9) the equation $q_{1}^{-2 \sigma+1}(\mathcal{L} v+R v)=0$ is written in the following form

$$
\begin{aligned}
& 2 \sigma\left(p_{2} \frac{\partial v}{\partial p_{2}}-s \frac{\partial v}{\partial s}\right)+2 \sigma t\left(t \frac{\partial v}{\partial t}-s \frac{\partial v}{\partial s}-2 p_{1} \frac{\partial v}{\partial p_{1}}\right) \\
+ & \left(-4 p_{1} \sigma s^{2} t^{2}+2 \sigma(2 \sigma-1) p_{2} t^{1-1 /(2 \sigma)} s\right) \frac{\partial v}{\partial p_{1}} \\
- & 4 p_{1} t^{1 /(2 \sigma)}\left(t+s^{2} t^{2}+1\right) s \frac{\partial v}{\partial p_{2}} \\
+ & 2 \sigma s^{2} t\left(s^{2} t^{2}+2 t+2\right)\left(t \frac{\partial v}{\partial t}-s \frac{\partial v}{\partial s}\right)=0 .
\end{aligned}
$$

For the sake of simplicity we write the right-hand side operators of (6.6) and (6.9) by $\mathcal{L}$ and $R$, respectively. Then (6.10) can be written in $\mathcal{L} v+R v=0$.

Now we will construct a transseries solution $v$ of (6.10) by the same method as in $\S 4$. Indeed, we replace the variables $q_{1}$ and $q_{2}$ with $t$ and $s$, respectively. The Lagrange-Charpit system corresponding to $\mathcal{L}$ in the new cordinates is given by

$$
\frac{d t}{t^{2}}=\frac{d s}{-s(1+t)}=\frac{d p_{1}}{-2 t p_{1}}=\frac{d p_{2}}{p_{2}} .
$$

Hence the transseries solution is given by

$$
v=\sum_{j=0}^{\infty} v_{j}\left(t, p_{1} t^{2}, p_{2} e^{1 / t}\right)\left(s t e^{-1 / t}\right)^{j},
$$

where $v_{0}\left(t, p_{1}^{0}, p_{2}^{0}\right),\left(p_{1}^{0}=p_{1} t^{2}, p_{2}^{0}=p_{2} e^{1 / t}\right)$ is a given polynomial of $p_{1}^{0}$ and $p_{2}^{0}$ with degree $N_{0} \geq 1$ which is independent of $t$ and $s$. We note that $\mathcal{L} v_{0}=0$. The $v_{j}$ 's are given by the formula like (4.10) which is obtained by inserting (6.12) into the equation $\mathcal{L} v+R v=0$ and by equating the coefficients of the powers of $s$. In order to determine $v_{j},(j \geq 1)$ we impose the initial conditions on $v_{j}$

$$
v_{j}\left(a_{0}, p_{1}^{0}, p_{2}^{0}\right) \equiv 0, \quad j=1,2, \ldots
$$

For $\nu \geq 1$ we define

$$
w=\sum_{j=0}^{\nu} v_{j}\left(t, p_{1} t^{2}, p_{2} e^{1 / t}\right)\left(s t e^{-1 / t}\right)^{j},
$$


and

$$
f_{\nu}\left(t, s, p_{1}, p_{2}\right):=\mathcal{L} w+R w .
$$

By definition $f_{\nu}\left(t, s, p_{1}, p_{2}\right)$ is a polynomial of $s, p_{1}$ and $p_{2}$ and is holomorphic in $t$ in $\mathbb{C} \backslash[0, \infty)$ and satisfies $f_{\nu}\left(t, s, p_{1}, p_{2}\right)=O\left(s^{\nu+1}\right)$, as $s \rightarrow 0$.

We define the path $\gamma(\pi)$ as follows; Let $0<\theta<\pi / 2$ be a number and $\rho_{0}>0$ be a small number. We start from the origin and go to the point $\rho_{0} e^{i \theta}$ along the straight line. Then we go to the point $\rho_{0} e^{2 \pi i-i \theta}$ along the circle with center at the origin and radius $\rho_{0}$ counterclockwise. Finally we return to the origin along the straight line. Let $K>0$ be a given constant, and define

$$
\Sigma:=\left\{\left(t, p_{1}, p_{2}\right) ;\left|p_{1}\right|<K\left|t^{2} e^{-1 / t}\right|,\left|p_{2}\right|<K\left|t^{2} e^{-1 / t}\right|, \quad \theta \leq \arg t \leq 2 \pi-\theta\right\} .
$$

Then we have

LEMMA 6.2. There exists $K>0$ such that the restriction of $f_{\nu}$ to $\Sigma,\left.f_{\nu}\left(t, s, p_{1}, p_{2}\right)\right|_{\Sigma}$ is a holomorphic function of $t$ in $\mathbb{C} \backslash[0, \infty)$, and it is continuous in $t$ on the path $\gamma(\pi)$ such that $\left.\lim _{t \rightarrow 0, t \in \gamma(\pi)} t^{-2} f_{\nu}\left(t, s, p_{1}, p_{2}\right)\right|_{\Sigma}=0$.

Proof. We note that $\mathcal{L}$ preserves the order of $s$. It follows that $\mathcal{L} w$ cancels with the corresponding terms in $R w$. Because $R w$ is the polynomial of $s$ with degree $\nu+4$, it is sufficient to show that restrictions to $\Sigma$ of terms of $R w$ are continuous in $t$ on the path $\gamma(\pi)$, and it has the desired estimate. In view of the definition of $R$ in (6.9) we have

$$
R w=\sum_{j}\left(R v_{j}\right)\left(s t e^{-1 / t}\right)^{j}+2 \sigma s^{2}\left(s^{2} t^{2}+2 t+2\right) \sum_{j} j v_{j}\left(s t e^{-1 / t}\right)^{j} .
$$

By definition $v_{0}=v_{0}\left(p_{1} t^{2}, p_{2} e^{1 / t}\right)$ is a polynomial of $p_{1}^{0}$ and $p_{2}^{0}$. Hence $v_{0}$ is holomorphic in $t$ in the domain $\mathbb{C} \backslash\{0\}$. If $\left(t, p_{1}, p_{2}\right) \in \Sigma$, then we easily see that $\left.\lim _{t \rightarrow 0, t \in \gamma(\pi)} v_{0}\right|_{\Sigma}=0$. Let $g_{1}(t)$ be the terms with degree 1 in $s$ of $R w$. Because $R w$ is divisable by $s, g_{1}(t)$ consists of terms with degree 1 in $s$ of $R v_{0}$. It follows that

$$
g_{1}(t)=2 \sigma(2 \sigma-1) t^{1-1 /(2 \sigma)} s p_{2} \frac{\partial v_{0}}{\partial p_{1}}-4 t^{1 /(2 \sigma)}(t+1) s p_{1} \frac{\partial v_{0}}{\partial p_{2}} .
$$

We can easily see that $g_{1}(t)$ is holomorphic in $\mathbb{C} \backslash[0, \infty)$. We want to show that there exists an $\varepsilon>0$ such that

$$
\left.\lim _{t \rightarrow 0, t \in \gamma(\pi)} t^{-2-\varepsilon} g_{1}(t)\right|_{\Sigma}=0 .
$$

Indeed, we recall that $v_{0}$ is a function of $p_{1}^{0}=p_{1} t^{2}$ and $p_{2}^{0}=p_{2} e^{1 / t}$. By (6.16) we have that for every $i_{k}, j_{k}=1,2\left(k=1,2, \ldots, N_{0}\right)$ with $N_{0}$ being the degree of $v_{0}$ the term

$$
\left.t^{-2}\left(\prod_{k=1}^{N_{0}} p_{i_{k}} \frac{\partial}{\partial p_{j_{k}}}\right) v_{0}\right|_{\Sigma}
$$

is bounded when $t \rightarrow 0, t \in \gamma(\pi)$. Hence (6.19) follows from (6.18) and (6.20). 
We compare the powers of $s$ in (6.10) with degree 1. By (6.11) and the construction of a transseries solution $v_{1}$ satisfies

$$
2 \sigma t^{2} \partial_{t} v_{1}\left(t, p_{1}^{0}, p_{2}^{0}\right)=t^{-1} e^{1 / t} g_{1}(t)
$$

where $p_{1}^{0}=p_{1} t^{2}$ and $p_{2}^{0}=p_{2} e^{1 / t}$. By integrating (6.21) we obtain

$$
v_{1}=v_{1}\left(t, p_{1}^{0}, p_{2}^{0}\right)=\frac{1}{2 \sigma} \int_{a_{0}}^{t} z^{-3} e^{1 / z} g_{1}(z) d z
$$

where $a_{0} \neq 0$. In case $t$ is close to the origin, then we first integrate from $a_{0}$ to some $a_{1} \neq 0$ on the straight line which connects the origin and $t$, then we integrate from $a_{1}$ to $t$ along the straight line.

Next we will show that

$$
\left.\lim _{t \rightarrow 0, t \in \gamma(\pi)} e^{-1 / t} v_{1}\right|_{\Sigma}=0 .
$$

Because $e^{-1 / t}$ tends to zero when $t \rightarrow 0, t \in \gamma(\pi)$, the integral from $a_{0}$ to $a_{1}$ in the integral (6.22) has no contribution when we calculate the limit of $\left.v_{1}\right|_{\Sigma}$ as $t \rightarrow 0, t \in$ $\gamma(\pi)$. Hence we consider

$$
e^{-1 / t} \int_{a_{1}}^{t} z^{-3} e^{1 / z} g_{1}(z) d z=\int_{a_{1}}^{(1+\delta) t} z^{-3} e^{1 / z-1 / t} g_{1}(z) d z+\int_{(1+\delta) t}^{t} z^{-3} e^{1 / z-1 / t} g_{1}(z) d z,
$$

where $\delta>0$ is a small positive constant.

We consider the first term in the right-hand side of (6.24). By setting $z=r e^{i \mu}$, $t=\rho e^{i \mu},(\rho<r)$ we have

$$
\operatorname{Re}\left(\frac{1}{z}-\frac{1}{t}\right)=-\left(\frac{1}{\rho}-\frac{1}{r}\right) \cos \mu \leq-\left(1-\frac{1}{1+\delta}\right) \frac{1}{\rho} \cos \mu=-\frac{\delta}{1+\delta} \frac{1}{\rho} \cos \mu,
$$

where $\cos \mu>0$. It follows that we have $\left|e^{1 / z-1 / t} z^{-3}\right| \leq K_{1}|t|$ for some $K_{1}>0$ independent of $t$ when $t \rightarrow 0, t \in \gamma(\pi)$. Hence we have

$$
\left|\int_{a_{1}}^{(1+\delta) t} z^{-3} e^{1 / z-1 / t} g_{1}(z) d z\right| \leq\left|\int_{\left|a_{1}\right|}^{(1+\delta) \rho} K_{1}\right| t|| g_{1}(z)|d| z|| .
$$

We restrict $p_{1}$ and $p_{2}$ to $\Sigma$. We note that $\left|p_{1} z^{2}\right| \leq\left|p_{1}\right|\left|a_{1}\right|^{2}<K\left|t^{2} e^{-1 / t}\right|\left|a_{1}\right|^{2}$ tends to 0 . Similarly, $\left|p_{2} e^{1 / z}\right|<K\left|t^{2} \exp (1 / z-1 / t)\right| \leq K|t|^{2}$ tends to 0. Hence the restriction to $\Sigma$ of the right-hand side tends to zero.

Next we will estimate the second term of the right-hand side of (6.24). Noting that $\left|e^{1 / z-1 / t}\right| \leq 1$ we will consider the integral

$$
\begin{aligned}
& \left|\int_{(1+\delta) \rho}^{\rho}\right| z^{-3} g_{1}(z)|d| z||=\left|\int_{(1+\delta) \rho}^{\rho}\right| z^{-1+\varepsilon}|| z^{-2-\varepsilon} g_{1}(z)|d| z|| \\
\leq & \max _{|t| \leq|z| \leq(1+\delta)|t|}\left|z^{-2-\varepsilon} g_{1}(z)\right|\left|\int_{(1+\delta) \rho}^{\rho}\right| z^{-1+\varepsilon}|d| z||,
\end{aligned}
$$


where $\varepsilon>0$. By (6.16) we see that the restriction of the right-hand side of (6.26) to $\Sigma$ tends to zero. This proves (6.23).

Let $g_{2}(t) \equiv g_{2}\left(t, p_{1}^{0}, p_{2}^{0}\right)$ be the terms with degree 2 in $s$ of $R w$. Clearly, $g_{2}$ appears from $R\left(v_{0}+v_{1} s t e^{-1 / t}\right)$. We define

$$
v_{2}=\frac{1}{2 \sigma} \int_{a_{0}}^{t} z^{-4} e^{2 / z} g_{2}(z) d z .
$$

Then we will show that

$$
\left.\lim _{t \rightarrow 0, t \in \gamma(\pi)} t^{2} e^{-2 / t} v_{2}\right|_{\Sigma}=0 .
$$

The terms with degree 2 appearing from $R v_{0}$ is given by $-4 p_{1} \sigma s^{2} t^{2} \partial_{p_{1}} v_{0}+4 \sigma s^{2}(t+$ 1) $t^{2} \partial_{t} v_{0}$. We consider the second term. We can easily see that the term with the largest growth in $v_{0}$ is given by $p_{2} e^{1 / t}$. Hence, in order to show (6.28) we may study the integral $p_{2} \int_{a_{1}}^{t} z^{-4} e^{3 / z} d z$. Because $\left|p_{2}\right| \leq\left|t^{2} e^{-1 / t}\right|$ on $\Sigma$, the restriction of the integral to $\Sigma$ satisfies (6.28).

We now consider remaining terms $\tilde{g}_{2}$ in $g_{2}$. We will show that there exists an $\varepsilon>0$ such that

$$
\left.\lim _{t \rightarrow 0, t \in \gamma(\pi)} t^{-2-\varepsilon} \tilde{g}_{2}(t)\right|_{\Sigma}=0 .
$$

Clearly, if we can show this, then we have (6.29) by the same argument as for $v_{1}$. By (6.20) the term $-4 p_{1} \sigma s^{2} t^{2} \partial_{p_{1}} v_{0}$ satisfies the estimate like (6.29). Next we consider terms with degree 2 in $s$ appearing from $R\left(v_{1} s t e^{-1 / t}\right)$

$$
\left(2 \sigma(2 \sigma-1) s t^{1-1 / 2 \sigma} p_{2} \partial_{p_{1}} v_{1}-4 s t^{1 / 2 \sigma}(t+1) p_{1} \partial_{p_{2}} v_{1}\right)\left(s t e^{-1 / t}\right) .
$$

In view of (6.18) and (6.22) we see that differentiations in (6.30) are estimated by

$$
\left.\left(p_{i_{1}} \partial_{p_{j_{1}}}\right)\left(p_{i_{2}} \partial_{p_{j_{2}}}\right) v_{0}\right|_{\Sigma}, i_{1}, i_{2}, j_{1}, j_{2}=1,2 \text {. }
$$

By (6.20) we see that $t^{-2}$ times of the terms in (6.31) are bounded. Hence we have (6.29).

We will show that

$$
\left.\lim _{t \rightarrow 0, t \in \gamma(\pi)} t^{3} e^{-3 / t} v_{3}\right|_{\Sigma}=0
$$

where

$$
v_{3}=\frac{1}{2 \sigma} \int_{a_{0}}^{t} z^{-5} e^{3 / z} g_{3}(z) d z,
$$

where $g_{3}(t)$ consists of terms with degree 3 in $s$ of $R w$. Clearly, $g_{3}(t)$ is equal to terms with degree 3 in $s$ of $R\left(v_{0}+\left(t s e^{-1 / t}\right) v_{1}+\left(t s e^{-1 / t}\right)^{2} v_{2}\right)$.

First we note that if the terms in $g_{3}$ satisfies an estimate like $\left.\tilde{g}_{2}\right|_{\Sigma}$ in (6.29), then the similar argument as for $v_{1}$ or $v_{2}$ shows that (6.32) holds. The terms with $s^{3}$ in $R v_{0}$ is given by $-4 p_{1} s^{3} t^{2+1 /(2 \sigma)}\left(\partial v_{0} / \partial p_{2}\right)$. The restriction of the term to $\Sigma$ has the similar estimate like $\left.g_{2}(t)\right|_{\Sigma}$ in (6.29). Next we will consider $R\left(\left(t s e^{-1 / t}\right) v_{1}\right)$. The terms with 
$s^{3}$ appear from $\left(R v_{1}\right)\left(t s e^{-1 / t}\right)$ and $-4 \sigma s^{3}(t+1) t e^{-1 / t} v_{1}$. As to $\left(R v_{1}\right)\left(t s e^{-1 / t}\right)$ we need to estimate

$$
-4 \sigma s^{3} t^{3} e^{-1 / t} p_{1} \partial_{p_{1}} v_{1}+4 \sigma s^{3}(t+1) e^{-1 / t} t^{3} \partial_{t} v_{1}
$$

The first term can be estimated by a similar method as in the above. Hence we consider the second term. Because $2 \sigma t^{2} \partial_{t} v_{1}=t^{-1} e^{1 / t} g_{1}(t)$, we see that the second term is equal to $2 s^{3}(t+1) g_{1}(t)$. The term has a similar estimate like (6.29).

Next we consider $-4 \sigma s^{3}(t+1) t e^{-1 / t} v_{1}$. In view of (6.33) we consider the integral

$$
\begin{aligned}
& \int_{a_{0}}^{t} z^{-4} e^{2 / z} v_{1}(z) d z=\int_{a_{0}}^{t}\left(-z^{-3} e^{2 / z}-\frac{1}{2}\left(z^{-2} e^{2 / z}\right)^{\prime}\right) v_{1}(z) d z \\
= & -\frac{1}{2} \int_{a_{0}}^{t}\left(z^{-2} e^{2 / z}\right)^{\prime} v_{1}(z) d z-\int_{a_{0}}^{t} z^{-3} e^{2 / z} v_{1}(z) d z .
\end{aligned}
$$

In order to estimate the first term of the right-hand side we consider

$$
\left.z^{-2} e^{2 / z} v_{1}(z)\right|_{a_{0}} ^{t}-\int_{a_{0}}^{t} z^{-2} e^{2 / z} v_{1}^{\prime}(z) d z
$$

By (6.23) $t^{3} e^{-3 / t}$ times the first term of the right-hand side of (6.35) restricted to $\Sigma$ tends to 0 when $t \rightarrow 0, t \in \gamma(\pi)$. In order to estimate the second term we consider, by $2 \sigma v_{1}^{\prime}(z)=z^{-3} e^{1 / z} g_{1}(z)$,

$$
\frac{1}{2 \sigma} \int_{a_{0}}^{t} z^{-5} e^{3 / z} g_{1}(z) d z
$$

Multiplying the term with $t^{3} e^{-3 / t}$ leads to

$$
\frac{1}{2 \sigma} \int_{a_{0}}^{t}(t / z)^{3} z^{-2} e^{3 / z-3 / t} g_{1}(z) d z
$$

Because one may assume $|t / z| \leq 1$, we are lead to the same estimate like (6.24). Hence $t^{3} e^{-3 / t}$ times the first term of the right-hand side of (6.34) restricted to $\Sigma$ tends to 0 when $t \rightarrow 0, t \in \gamma(\pi)$. We note that in the second term of the right-hand side of (6.34) the negative power in the integrand is improved compared to the lefthand side term by partial integration. By (6.23) $t^{3} e^{-3 / t}$ times of the restriction to $\Sigma$ of the second term of the right-hand side of (6.34) tends to zero because $|z / t| \leq 1$, $|\exp (3 / z-3 / t)| \leq 1$. This completes the estimate of $R\left(\left(t s e^{-1 / t}\right) v_{1}\right)$.

Next we will consider $s^{3}$ terms in $R\left(\left(t s e^{-1 / t}\right)^{2} v_{2}\right)$. Clearly we may consider $\left(t s e^{-1 / t}\right)^{2} R v_{2}$. The terms with degree 3 in $s$ are given by

$$
2 \sigma(2 \sigma-1) e^{-2 / t} t^{3-1 /(2 \sigma)} s^{3} p_{2} \frac{\partial v_{2}}{\partial p_{1}}-4 t^{2+1 /(2 \sigma)} e^{-2 / t}(t+1) s^{3} p_{1} \frac{\partial v_{2}}{\partial p_{2}} .
$$

Inserting the formula of $v_{2}$ we are lead to the estimate of $v_{0}$. It is estimated by (6.20). Hence we see that $R\left(\left(t s e^{-1 / t}\right)^{2} v_{2}\right)$ have the estimate like (6.29). This proves (6.32).

We proceed by induction and show that for $\nu=2,3, \ldots$,

$$
\left.\lim _{t \rightarrow 0, t \in \gamma(\pi)} t^{\nu} e^{-\nu / t} v_{\nu}\right|_{\Sigma}=0
$$


Indeed, the estimates of differentiations with respect to $p_{j}$ 's are reduced to (6.20). The differentiations with respect to $t$ are estimated via partial integration and the argument as for $v_{1}, v_{2}$ and $v_{3}$. Moreover, if we denote by $g_{\nu+1}$ the terms with degree $\nu$ in $s$ in $\mathcal{L} w+R w, w=\sum_{j=0}^{\nu} v_{j}\left(s t e^{-1 / t}\right)^{j}$, then

$$
\left.\lim _{t \rightarrow 0, t \in \gamma(\pi)} t^{-2-\varepsilon} g_{\nu+1}(t)\right|_{\Sigma}=0
$$

As to the terms with degree $>\nu+1$ in $s$ of $\mathcal{L} w+R w, w=\sum_{j=0}^{\nu} v_{j}\left(s t e^{-1 / t}\right)^{j}$ we can estimate the terms by the same argument as for the estimate of $g_{j}$ 's. This proves the desired estimate in Lemma 6.2. This ends the proof.

We use the partial Borel transform with respect to $t$

$$
g(\zeta) \equiv(\mathcal{B} f)(\zeta)=\frac{1}{2 \pi i} \int_{\gamma(\pi)} t f(t) \exp (\zeta / t) d t^{-1},
$$

where $\zeta$ is the dual variable of $t$ with respect to the Borel transform.

We assume that $f(t)$ in $(6.41)$ is analytic in the sectorial domain bounded by $\gamma(\pi)$ and continuous up to the boundary. The inverse Borel transform of $g(\zeta)$ is given by the Laplace integral

$$
\left(\mathcal{B}^{-1} g\right)(t)=t^{-1} \int_{0}^{\infty(\tau)} g(\zeta) \exp (-\zeta / t) d \zeta(=f(t)) .
$$

The integration in (6.42) is taken along the ray which starts from the origin and goes to infinity in the direction $\tau$ which is sufficiently close to $\pi$.

We introduce a function space. Let $\varepsilon_{0}>0$ be a given small constant and $N_{0}$ be a positive integer. We define

$$
\Gamma_{0}:=\left\{\zeta \in \mathbb{C} ;|\zeta|<\varepsilon_{0} \text { or }|\arg \zeta-\pi|<\varepsilon_{0}\right\} .
$$

Let $\Omega_{0}$ and $\Omega$ be defined by

$$
\begin{gathered}
\Omega_{0}=\left\{\left(\zeta, p_{1}, p_{2}\right) \in \mathbb{C}^{3} ; \zeta \in \Gamma_{0},\left|p_{1}\right|<\varepsilon_{0},\left|p_{2}\right|<\varepsilon_{0}\right\}, \\
\Omega:=\left\{s \in \mathbb{C} ;|s|<\varepsilon_{0}\right\} \times \Omega_{0} .
\end{gathered}
$$

We denote by $H(\Omega)$ the set of functions $w$ which are polynomials of $p_{1}$ and $p_{2}$ with degree at most $N_{0}$, holomorphic in $\Omega$ and continuous up to the boundary such that there exist constants $c>0$ and $K>0$ for which

$$
\left|w\left(s, \zeta, p_{1}, p_{2}\right)\right| \leq K|\exp (c|\zeta|)|, \quad \forall\left(s, \zeta, p_{1}, p_{2}\right) \in \Omega .
$$

We define the norm $\|w\|$ of $w$ as the infimum of $K$ satisfying (6.46). The space $H(\Omega)$ is a Banach space with the norm $\|\cdot\|$.

We recall the following formula

$$
\mathcal{B}\left(t^{2} \partial_{t} f\right)(\zeta)=\zeta(\mathcal{B} f)(\zeta)-\mathcal{B}(t f)(\zeta)
$$

The formula is proved by the direct computations of the Borel transform. 
Let $\lambda$ be a positive number. We denote by $\alpha_{\lambda} \equiv \alpha_{\lambda}(\zeta, D)$ the Borel transform of the multiplication operator $f(t) \mapsto t^{\lambda} f(t)$. Namely, $\alpha_{\lambda}$ is given by $\mathcal{B}\left(t^{\lambda} f\right)=\alpha_{\lambda} \mathcal{B}(f)$.

LEMma 6.3. There exists a constant $K_{0}>0$ such that the Borel transform of the operator $f \mapsto t^{\lambda} f(\lambda>0)$ has the estimate

$$
\left\|\mathcal{B}\left(t^{\lambda} f\right)\right\| \leq K_{0}\|\mathcal{B}(f)\|
$$

for every $f$ holomorphic in $\gamma(\pi)$ and continuous up to the boundary such that $\mathcal{B}(f) \in$ $H(\Omega)$. Moreover, the constant $K_{0}>0$ can be chosen arbitrarily small if we take $c$ in (6.46) sufficiently large.

Proof. For the sake of simplicity we write $H:=H(\Omega)$. We recall that the Borel transform of $t^{\lambda}$ is equal to $\zeta^{\lambda} / \Gamma(1+\lambda)$. Since $\hat{f}:=\mathcal{B}(f) \in H$ we have the formula

$$
\mathcal{B}\left(t^{\lambda} f\right)(\zeta)=\frac{1}{\Gamma(1+\lambda)} \frac{d}{d \zeta} \int_{0}^{\zeta}(\zeta-\eta)^{\lambda} \hat{f}(\eta) d \eta=\frac{1}{\Gamma(\lambda)} \int_{0}^{\zeta}(\zeta-\eta)^{\lambda-1} \hat{f}(\eta) d \eta
$$

where the integral is taken along the straight line in the sector $\Gamma_{0}$ which connects the origin and $\zeta \in \Gamma_{0}$.

We first consider the case $0<\lambda<1$. Let $\theta=\arg \zeta$ and $\varepsilon>0$ be a small number chosen later. Then the $\Gamma(\lambda)$-times of the right-hand side of (6.49) can be written in the following form

$$
\int_{0}^{\zeta-\varepsilon e^{i \theta}}(\zeta-\eta)^{\lambda-1} \hat{f}(\eta) d \eta+\int_{\zeta-\varepsilon e^{i \theta}}^{\zeta}(\zeta-\eta)^{\lambda-1} \hat{f}(\eta) d \eta \equiv I_{1}+I_{2}
$$

We estimate $I_{2}$. By the definition of the integral we have

$$
\begin{gathered}
\left|I_{2}\right| \leq \int_{|\zeta|-\varepsilon}^{|\zeta|}|\zeta-\eta|^{\lambda-1}|\hat{f}(\eta)| d|\eta| \leq\|\hat{f}\| \int_{|\zeta|-\varepsilon}^{|\zeta|}(|\zeta|-|\eta|)^{\lambda-1} e^{c|\eta|} d|\eta| \\
\leq\|\hat{f}\| e^{c|\zeta|} \int_{|\zeta|-\varepsilon}^{|\zeta|}(|\zeta|-|\eta|)^{\lambda-1} d|\eta|=\|\hat{f}\| e^{c|\zeta|} \int_{0}^{\varepsilon} s^{\lambda-1} d s=\|\hat{f}\| e^{c|\zeta|} \varepsilon^{\lambda} / \lambda .
\end{gathered}
$$

As to $I_{1}$ we can similarly estimate

$$
\left|I_{1}\right| \leq \int_{0}^{|\zeta|-\varepsilon}|\zeta-\eta|^{\lambda-1}|\hat{f}(\eta)| d|\eta| \leq\|\hat{f}\| \int_{0}^{|\zeta|-\varepsilon}(|\zeta|-|\eta|)^{\lambda-1} e^{c|\eta|} d|\eta| .
$$

Because $|\zeta|-|\eta| \geq \varepsilon$ and $0<\lambda<1$, we have $(|\zeta|-|\eta|)^{\lambda-1} \leq \varepsilon^{\lambda-1}$. It follows that the right-hand side of (6.52) can be estimated by

$$
\|\hat{f}\| \varepsilon^{\lambda-1} \int_{0}^{|\zeta|-\varepsilon} e^{c|\eta|} d|\eta| \leq \frac{\|\hat{f}\| \varepsilon^{\lambda}}{c \varepsilon} e^{c|\zeta|-c \varepsilon}
$$

Therefore we have

$$
\left|\mathcal{B}\left(t^{\lambda} f\right)(\zeta)\right| \leq\|\hat{f}\| e^{c|\zeta|} \varepsilon^{\lambda}\left(\lambda^{-1}+\left(c \varepsilon e^{c \varepsilon}\right)^{-1}\right) / \Gamma(\lambda) .
$$

We take $\varepsilon>0$ sufficiently small, then $c>0$ sufficiently large. We can make the constant $K_{0}=\varepsilon^{\lambda}\left(\lambda^{-1}+\left(c \varepsilon e^{c \varepsilon}\right)^{-1}\right) / \Gamma(\lambda)$ arbitrarily small. This proves (6.48). 
Next we consider the case $\lambda \geq 1$. We see that $\Gamma(\lambda) \mathcal{B}\left(t^{\lambda} f\right)(\zeta)$ is given by the integral in the right-hand side of (6.49). By similar calculations as in the case $0<\lambda<1$, we see that it is estimated by

$$
\|\hat{f}\| \int_{0}^{|\zeta|}(|\zeta|-|\eta|)^{\lambda-1} e^{c|\eta|} d|\eta| .
$$

If $\lambda=1$, then the integral (6.55) is bounded by $\|\hat{f}\| e^{c|\zeta|} / c$. In the case $\lambda>1$, by the partial integration it is equal to

$$
\begin{aligned}
& \|\hat{f}\|\left(\left[\frac{e^{c s}}{c}(|\zeta|-s)^{\lambda-1}\right]_{s=0}^{s=|\zeta|}+\frac{\lambda-1}{c} \int_{0}^{|\zeta|}(|\zeta|-|\eta|)^{\lambda-2} e^{c|\eta|} d|\eta|\right) \\
& \leq\|\hat{f}\|\left(-\frac{1}{c}|\zeta|^{\lambda-1}+\frac{\lambda-1}{c} \int_{0}^{|\zeta|}(|\zeta|-|\eta|)^{\lambda-2} e^{c|\eta|} d|\eta|\right) \\
& \leq\|\hat{f}\| \frac{\lambda-1}{c} \int_{0}^{|\zeta|}(|\zeta|-|\eta|)^{\lambda-2} e^{c|\eta|} d|\eta| .
\end{aligned}
$$

This implies that the estimate of our integral is reduced to that of the same integral with $\lambda$ replaced by $\lambda-1$. Hence, by the inductive argument we have the same estimate. This proves the lemma.

Proof of Theorem 6.1.

Let $\nu$ be a positive integer chosen later and define $w$ by (6.14). Let $u$ the solution of (6.10). If we set $u=w+v, v=O\left(s^{\nu+1}\right)$, then $v$ satisfies the equation

$$
\mathcal{L} v+R v=-\mathcal{L} w-R w \equiv f
$$

where $f=O\left(s^{\nu+1}\right)$. Let $\gamma(\pi)$ be the path given in Lemma 6.2. We use the coordinate $(t, s)$. The function $f$ is holomorphic in $\mathbb{C} \backslash[0, \infty)$, continuous on $\gamma(\pi)$, and satisfies $f=O\left(s^{\nu+1}\right)$ by Lemma 6.2. In order to construct the solution in $\Sigma \times\left\{|s|<\varepsilon_{1}\right\}$ we make the change of the variables $p_{1}=\tilde{p}_{1} t^{2} e^{-1 / t}, p_{2}=\tilde{p}_{2} t^{2} e^{-1 / t}$. Clearly, for $\left(t, p_{1}, p_{2}\right) \in \Sigma$ we have $\left|\tilde{p}_{j}\right|<K(j=1,2)$ and vice versa. Then we can easiy verify that $p_{i} \frac{\partial}{\partial p_{j}}=\tilde{p}_{i} \frac{\partial}{\partial \tilde{p}_{j}}$ for $j=1,2$. It follows that in the equation (6.10) we may replace $p_{j}$ with $\tilde{p}_{j}$. For the sake of simplicity we denote the variable $\tilde{p}_{j}$ with $p_{j}$ in (6.10). We note that we may think that $p_{j}$ moves in some neighborhood of the origin.

We set $g:=s^{-\nu-1} f$ and $V:=s^{-\nu-1} v$. By taking the partial Borel transform with respect to $t$, we have

$$
\left(\hat{\mathcal{L}}-2 \sigma\left(1+\alpha_{1}\right)(\nu+1)\right) \hat{V}+\hat{R} \hat{V}=\hat{g}
$$


where $\hat{g}=\mathcal{B}(g), \hat{V}:=\mathcal{B}(V)$ and

$$
\begin{aligned}
\hat{\mathcal{L}} \hat{V} & :=2 \sigma\left(p_{2} \frac{\partial}{\partial p_{2}}-\left(1+\alpha_{1}\right) s \frac{\partial}{\partial s}+\zeta-\alpha_{1}-2 \alpha_{1} p_{1} \frac{\partial}{\partial p_{1}}\right) \hat{V} \\
\hat{R} \hat{V} & :=\left(-4 p_{1} \sigma s^{2} \alpha_{2}+2 \sigma(2 \sigma-1) p_{2} s \alpha_{1-1 /(2 \sigma)}\right) \frac{\partial \hat{V}}{\partial p_{1}} \\
& -4 p_{1} s\left(\alpha_{1+1 /(2 \sigma)}+s^{2} \alpha_{2+1 /(2 \sigma)}+\alpha_{1 /(2 \sigma)}\right) \frac{\partial \hat{V}}{\partial p_{2}} \\
& +2 \sigma s^{2}\left(s^{2} \alpha_{2}+2 \alpha_{1}+2\right)\left(\zeta-\alpha_{1}\left(1+s \frac{\partial}{\partial s}\right)\right) \hat{V} .
\end{aligned}
$$

Here we used the fact that the Borel transform of $s^{2} t\left(s^{2} t^{2}+2 t+2\right)\left(t \frac{\partial u}{\partial t}-s \frac{\partial u}{\partial s}\right)$ is given by

$$
s^{2}\left(s^{2} \alpha_{2}+2 \alpha_{1}+2\right) \mathcal{B}\left(t^{2} \frac{\partial u}{\partial t}-s t \frac{\partial u}{\partial s}\right)=s^{2}\left(s^{2} \alpha_{2}+2 \alpha_{1}+2\right)\left(\zeta-\alpha_{1}\left(1+s \frac{\partial}{\partial s}\right)\right) \hat{u} .
$$

We expand $\hat{g}$ in the power series of $s, \hat{g}=\sum_{k=0}^{\infty} \hat{g}_{k}\left(\zeta, p_{1}, p_{2}\right) s^{k}$ and we look for the solution $\hat{V}$ of (6.58) in the form

$$
\hat{V}=\sum_{k=0}^{\infty} \hat{V}_{k}\left(\zeta, p_{1}, p_{2}\right) s^{k} .
$$

By inserting these expansions into (6.58) we have the recurrence formula

$$
\begin{aligned}
& \left(\hat{\mathcal{L}}_{0}-2 \sigma(\nu+1+k)\left(1+\alpha_{1}\right)\right) \hat{V}_{k}=\hat{g}_{k} \\
- & 4 \sigma \alpha_{2} p_{1} \frac{\partial}{\partial p_{1}} \hat{V}_{k-2}+2 \sigma(2 \sigma-1) \alpha_{1-1 /(2 \sigma)} p_{2} \frac{\partial}{\partial p_{1}} \hat{V}_{k-1} \\
- & 4\left(\alpha_{1+1 /(2 \sigma)}+\alpha_{1 /(2 \sigma)}\right) p_{1} \frac{\partial}{\partial p_{2}} \hat{V}_{k-1}-4 \alpha_{2+1 /(2 \sigma)} p_{1} \frac{\partial}{\partial p_{2}} \hat{V}_{k-3} \\
+ & 2 \sigma \alpha_{2}\left(\zeta-\alpha_{1}(k-3)\right) \hat{V}_{k-4}+4 \sigma\left(\alpha_{1}+1\right)\left(\zeta-\alpha_{1}(k-1)\right) \hat{V}_{k-2},
\end{aligned}
$$

where $k=0,1, \ldots$ and

$$
\hat{\mathcal{L}}_{0}=\hat{\mathcal{L}}+2 \sigma\left(1+\alpha_{1}\right) s \frac{\partial}{\partial s}
$$

In the following $H\left(\Omega_{0}\right)$ denotes the set of holomorphic functions $w\left(\zeta, p_{1}, p_{2}\right)$ being holomorphic in $\Omega_{0}$ and polynomials of $p_{1}$ and $p_{2}$ with degree at most $N_{0}$. We equip $H\left(\Omega_{0}\right)$ with the norm similar to $H(\Omega)$. We shall show that there exists $\nu$ such that $\hat{\mathcal{L}}_{0}-2 \sigma(\nu+1+k)\left(1+\alpha_{1}\right)$ on $H\left(\Omega_{0}\right)$ is invertible when $k=0,1,2, \ldots$ Because a function in $H\left(\Omega_{0}\right)$ is a polynomial of $p_{1}$ and $p_{2}$ with degree at most $N_{0}$, the operators $p_{j}\left(\partial / \partial p_{j}\right)$ in $\hat{\mathcal{L}}_{0}$ are bounded continuous operators on $H\left(\Omega_{0}\right)$. Because the operators $\alpha_{\lambda}$ is a bounded continuous operator whose norm can be made arbitrarily small by Lemma 6.3 , it follows that $\hat{\mathcal{L}}_{0}-2 \sigma(\nu+1+k)\left(1+\alpha_{1}\right)$ is invertible for $\zeta \in \Gamma_{0}$ if we 
take $\nu$ sufficiently large. Moreover, by the Neumann series we can easily show that there exists $K_{1}>0$ such that, for $k=0,1,2, \ldots$ and all $\zeta \in \Gamma_{0}$

$$
\left\|\left(\hat{\mathcal{L}}_{0}-2 \sigma(\nu+1+k)\left(1+\alpha_{1}\right)\right)^{-1}\right\| \leq \frac{K_{1}}{1+k+|\zeta|} .
$$

It follows that if we operate $\left(\hat{\mathcal{L}}_{0}-2 \sigma(\nu+1+k)\left(1+\alpha_{1}\right)\right)^{-1}$ to $(6.60)$, then the right-hand side terms in (6.60) are bounded by some constants which can be taken arbitrarily small if $\varepsilon_{0}$ is sufficiently small for all $\zeta \in \Gamma_{0}$ and $k=0,1,2 \ldots$ It follows that the $V_{k}$ 's can be determined recursively from (6.60) in $H\left(\Omega_{0}\right)$. Moreover, there exist $K_{2}>0$ and $C_{0}>0$ such that we have the estimate

$$
\left\|V_{k}\right\| \leq K_{2} C_{0}^{k}, \quad k=0,1,2, \ldots
$$

This proves that the series (6.59) converges in $H(\Omega)$. If we make the partial Laplace transform with respect to $\zeta$ in (6.58), then we have the solution $U$ as in Theorem 6.1. This ends the proof.

\section{REFERENCES}

[1] Gorni G. and Zampieri G.: Analytic-non-integrability of an integrable analytic Hamiltonian system, Differential Geometry $\mathcal{G}$ its Appl., 22, 287-296 (2005).

[2] Ito H.: Integrability of Hamiltonian systems and Birkoff normal forms in the simple resonance case, Math. Ann. 292, 411-444 (1992).

[3] Stolovitch L.: Singular complete integrability, Publ. Math. I.H.E.S., 91, 134-210 (2000).

[4] Yoshino M.: Analytic non-integrable Hamiltonian systems and irregular singularity, Annali di matematica pura ed applicata, 187, 555-562 (2008). 